Review

\title{
Advances in nutraceutical delivery systems: From formulation design for bioavailability enhancement to efficacy and safety evaluation
}

\author{
Raquel F.S. Gonçalves ${ }^{\mathrm{a}}$, Joana T. Martins ${ }^{\mathrm{a}}$, Catarina M.M. Duarte ${ }^{\mathrm{b}, \mathrm{c}}$, António A. Vicente ${ }^{\mathrm{a}}$, \\ Ana C. Pinheiro ${ }^{\mathrm{a}, \mathrm{b}, *}$ \\ ${ }^{\text {a }}$ CEB - Centre of Biological Engineering, University of Minho, 4710-057 Braga, Portugal \\ ${ }^{\mathrm{b}}$ Instituto de Biologia Experimental e Tecnológica, Avenida da República, Quinta-do-Marquês, Estação Agronómica Nacional, Apartado 12, 2781-901 Oeiras, Portugal \\ ${ }^{c}$ Instituto de Tecnologia Química e Biológica António Xavier, Universidade Nova de Lisboa, Av. da República, 2780-157 Oeiras, Portugal
}

\section{A R T I C L E I N F O}

\section{Keywords:}

Nano-delivery systems

Food bioactive compounds

Gastrointestinal fate evaluation

Bioaccessibility

Toxicity

\begin{abstract}
A B S T R A C T
Background: Aiming at the enhancement of food products' nutritional and health value, the incorporation of nutraceuticals has attracted increasing interest in the last years. However, they often exhibit low water solubility and stability, limiting their direct incorporation into food products. Also, they show very low bioavailability due to limited bioaccessibility, poor absorption and/or chemical transformation within the gastrointestinal tract. This renders their health benefits extremely difficult to be realized by the consumers.

Scope and approach: In the present review the recent innovations regarding the formulation and design of biobased micro and nano-delivery systems to encapsulate nutraceuticals is discussed; it also gives an overview of the challenges associated to their development; and highlights some strategies to enhance nutraceuticals' bioavailability. An insight about delivery systems' potential toxicity (in particular at nano-scale) is also provided. Key findings and conclusions: Recent developments in the design of bio-based delivery systems offer the possibility of stabilizing and enhancing nutraceuticals' functionality within food products. In fact, different strategies can be used to enhance nutraceuticals' bioavailability: i) nano-delivery systems, besides showing a huge potential for the protection of valuable nutraceuticals during food processing/digestion, can be used to increase their bioavailability; ii) absorption enhancement technologies have been successfully used to increase nutraceuticals' membrane permeation; and iii) excipient foods have been shown to improve nutraceuticals' biological activity. However, the application of these enabling technologies to food is hindered by very pertinent issues that can be summarized in the effective preservation/maximization of the nutraceuticals' bioactivity and safety, once inside the human body.
\end{abstract}

\section{Introduction}

In the age of chronic diseases (obesity, Type II diabetes and cardiovascular diseases) and rising number of elderly people, bioactive compounds that exhibit health-promoting effects are receiving increasing attention. Since the strong link between dietary habits and health has been established, food, in addition of being a good source of nutrients with good sensory appeal, also needs to be beneficial to the health and well-being of the consumers. However, in the modern lifestyle it could be difficult to ingest all of the nutrients needed to maintain normal body functions or promote good health. The incorporation of nutraceuticals in food products provides a simple way of developing novel functional foods. In fact, there is an increasing interest in the development of functional foods/beverages enriched with different nutraceuticals. Nutraceuticals can be defined as nutritional components that provide therapeutic or physiological benefits beyond the basic nutritional needs and include a wide range of compounds such as bioactive peptides, phenolic compounds, carotenoids, lipids, vitamins, etc. (Ting, Jiang, Ho, \& Huang, 2014). However, the effectiveness of nutraceuticals in providing therapeutic or physiologic benefits greatly depends on preserving their bioavailability, defined as the fraction of an ingested compound that is absorbed and available for physiological functions (i.e. reaches the systemic circulation in an active form) (Ting et al., 2014). Different factors can compromise the bioavailability of a compound, including an insufficient gastric residence time, low permeability and/or solubility within the gastrointestinal (GI) tract and instability during food processing/storage or in GI tract (Leonard, 2000). In fact, most of nutraceuticals are unlikely to provide the intended bioactive properties without the use of an appropriate delivery system. This system should be designed to overcome the specific factors

\footnotetext{
* Corresponding author. CEB - Centre of Biological Engineering, University of Minho, 4710-057 Braga, Portugal.

E-mail address: anapinheiro@deb.uminho.pt (A.C. Pinheiro).
} 


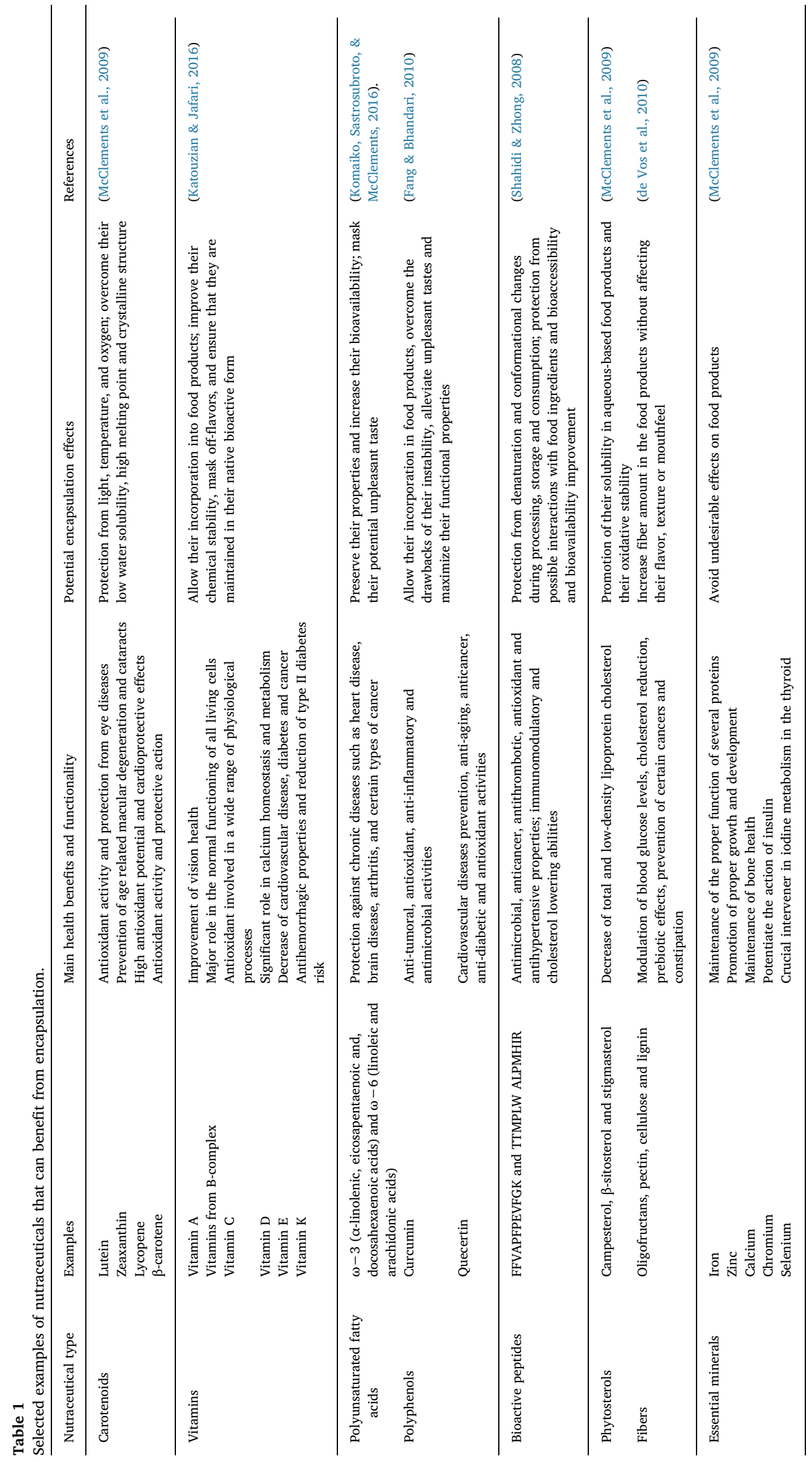


affecting the loaded nutraceutical functionality. Carotenoids, vitamins, fatty acids, polyphenols, bioactive peptides, phytosterols, fibers and essential minerals are some examples of nutraceuticals with claimed biological activity that can benefit from incorporation into delivery systems (Table 1).

The main challenges of incorporating nutraceuticals such as $\beta$-carotene, curcumin, vitamins $\mathrm{A}, \mathrm{D}, \mathrm{E}$ and $\mathrm{K}$ in food products are their chemical instability during food processing/storage (e.g. sensibility to light, oxygen, heat) or within GI tract (e.g. easily degraded by enzymes and/or $\mathrm{pH}$ ), their low water solubility and low bioavailability. All these factors can affect nutraceuticals' functionality, and consequently, their health benefits may not be recognized even when ingested in high amounts. Furthermore, when directly incorporated in food products, nutraceuticals with unpleasant sensory properties (e.g. polyunsaturated fatty acids) can negatively affect foods' properties and shelf-life. Alternatively, some of those compounds (e.g. vitamin C) can have undesirable interactions with other food components, negatively affecting foods' appearance, texture, mouthfeel, stability and bioavailability of important components. Consequently, encapsulation of nutraceuticals is often required to i) protect them against adverse external factors; ii) allow their incorporation into food products (e.g. increase the lipophilic compounds' solubility/dispensability in water-based environments), iii) mask any off-flavors; iv) allow their controlled release; and v) preserve/ maximize their functional properties, i.e. to ensure that they are maintained in their native bioactive form until they reach the site of action. Therefore, delivery systems at micro and nano-scale have attracted considerable interest worldwide over the past years. Also, in recent years, different strategies have been used and significant advances have been made in the area of delivery systems to enhance the bioavailability and consequently, nutraceuticals' efficacy.

This work presents a comprehensive state-of-the-art review of the recent advances in the development of micro and nano-scale delivery systems to improve the bioavailability of nutraceuticals, from their formulation/design to the evaluation of their efficacy. Potential risks that may arise from their use (in particular at nano-scale) will be also addressed.

\section{Formulation and design of delivery systems for nutraceuticals}

The successful development of delivery systems for nutraceuticals requires the knowledge of their properties and the use of adequate materials and production techniques. In fact, the choice of the adequate encapsulation procedure is a key step once many nutraceuticals are sensitive to heat and to high temperature during the encapsulation process, which could cause loss of their bioactivity.

The design of delivery systems for nutraceuticals that have adequate physical and chemical stability as well as food-grade status, cost effectiveness and technological feasibility can involve numerous challenges. Moreover, to obtain a satisfactory bioavailability for such nutraceuticals is even a more challenging task.

\subsection{Desirable characteristics of delivery systems}

Being one of the delivery systems' main goals the improvement of nutraceuticals' bioavailability, those should be developed to promote nutraceuticals' aqueous solubility, adequate residence time in the GI tract, absorption across the intestinal line and stability to changing physiological environments (Ting et al., 2014). During the development or selection of a delivery system, it is important and useful to consider some parameters. The most relevant parameters are summarized in Fig. 1 and briefly described below:

\subsubsection{Food grade status}

In contrast to pharmaceutical products, the delivery systems applied in food products cannot be composed of synthetic chemical polymers due to the side effects that these can cause and due to the fact of being

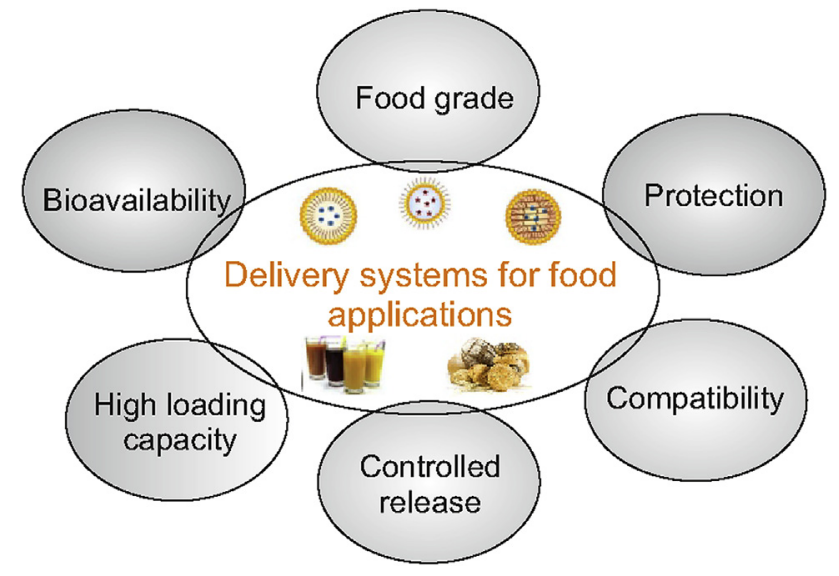

Fig. 1. Main desirable characteristics of delivery systems for food applications.

integrated in foods, there is no control over the amounts that consumers may wish to intake. Therefore, regulatory requirements also offer challenges to the development of delivery systems for nutraceuticals, once only food grade materials must be used for encapsulation or as core ingredients. These materials can be obtained from natural sources (i.e. bio-based materials), which is the case of proteins, starch, lipids, gums and cellulosic materials, or can be materials with Generally Recognized as Safe (GRAS) status such as cyclodextrins, low-molecular weight emulsifiers (e.g. Tweens) and mineral salts (Augustin \& Sanguansri, 2008). Moreover, the processing operations must also have regulatory approval in the country where the food will be sold (McClements, 2012a). Different countries have different regulations, for example the main governmental agencies regulating food safety are the European Food Safety Authority (EFSA) in Europe and the Food and Drug Administration (FDA) in the USA.

\subsubsection{Protection of the encapsulated nutraceutical}

Delivery systems should be designed to protect nutraceuticals during processing, storage and transport from adverse factors such as undesirable interactions with other food ingredients, $\mathrm{pH}$, light, temperature or oxygen. Furthermore, these systems should also protect the nutraceuticals from the severe GI environment (e.g. high activity of digestive enzymes and harsh acidic conditions in stomach) (McClements, Decker, Park, \& Weiss, 2009).

\subsubsection{Compatibility with the food matrix}

Food matrix is the first and major factor that affects the biological fate of the encapsulated nutraceutical. For example, the interactions between mixed micelles and compounds of the partially digested food matrix can provoke changes in the properties of the mixed micelles and alter the digestion and absorption of the nutraceuticals encapsulated inside them (Yao, Xiao, \& McClements, 2014). Additionally, delivery systems should be compatible with the food matrix, not affecting the properties of the food product (e.g. aroma, taste, appearance and texture). Delivery systems can be incorporated within different matrices: i) suspended in aqueous solution to be incorporated in beverages and drinks, ii) trapped inside a biopolymer matrix for incorporation in desserts, yogurts and sauces or iii) stuck within a solid matrix for incorporation in powders and cereal products (Yao et al., 2014). In the case of clear beverages, it is possible to use for example, nanoemulsions because the particles are so small that they do not strongly disperse the light (McClements et al., 2009).

\subsubsection{Controlled release capacity}

Another desirable characteristic is the controlled release ability, which consists in releasing the encapsulated compound with a specific concentration/time profile at the desirable site of action. The release 
Table 2

Most commonly used bio-based encapsulating materials.

\begin{tabular}{|c|c|c|c|}
\hline Encapsulating material & Examples & Attractive characteristics & References \\
\hline \multirow[t]{5}{*}{ Polysaccharides } & Starch & Inexpensive; easily available; form glassy solids after dehydration & (Panyoyai, Shanks, \& Kasapis, 2017) \\
\hline & Chitosan & $\begin{array}{l}\text { Abundant resource; cationic; antimicrobial; mucoadhesive; natural } \\
\text { swelling-deswelling behavior at different pHs; permeation enhancing } \\
\text { effect }\end{array}$ & $\begin{array}{l}\text { (Sotelo-Boyás, Correa-Pacheco, Bautista- } \\
\text { Baños, \& Corona-Rangel, 2017) }\end{array}$ \\
\hline & Alginate & Anionic; mucoadhesive; gelation properties & $\begin{array}{l}\text { (Paques, van der Linden, van Rijn, \& Sagis, } \\
\text { 2014) }\end{array}$ \\
\hline & Carrageenan & Anionic; gelling and film-forming capacities & (Li, Ni, Shao, \& Mao, 2014) \\
\hline & Pectin & Anionic; gelation and emulsification properties; anti-oxidative effect & (Noh et al., 2018) \\
\hline \multirow[t]{4}{*}{ Proteins } & $\beta$-lactoglobulin & $\begin{array}{l}\text { Inexpensive; ability to form gels; antioxidant activity; resistant to } \\
\text { gastric digestion }\end{array}$ & (Madalena et al., 2016) \\
\hline & Sodium Caseinate & Emulsification and gelation properties & $\begin{array}{l}\text { (Montes de Oca-Ávalos, Candal, \& } \\
\text { Herrera, 2017) }\end{array}$ \\
\hline & Lactoferrin & $\begin{array}{l}\text { Antibacterial; antiviral; immunomodulatory capacity; high iron } \\
\text { binding capacity; high isoelectric point }\end{array}$ & (Martins et al., 2016) \\
\hline & Zein & $\begin{array}{l}\text { Ability to self-assemble; insolubility in aqueous conditions; resistance } \\
\text { to gastric digestion }\end{array}$ & (Zhang, Cui et al., 2016) \\
\hline \multirow[t]{3}{*}{ Lipids } & $\begin{array}{l}\text { Long chain triglycerides (LCT) } \\
\text { (e.g. corn oil) }\end{array}$ & Increase bioaccessibility of lipophilic bioactive compounds & (Qian, Decker, Xiao, \& McClements, 2012) \\
\hline & $\begin{array}{l}\text { Medium chain triglycerides } \\
\text { (MCT) (e.g. Miglyol) }\end{array}$ & Neutral flavor profile & $\begin{array}{l}\text { (Walker, Gumus, Decker, \& McClements, } \\
\text { 2017) }\end{array}$ \\
\hline & Flavor oils (e.g. lemon oil) & $\begin{array}{l}\text { Strong antimicrobial and antioxidant activities; mask off-flavors; } \\
\text { typically present low rates and extents of digestion }\end{array}$ & (Walker et al., 2017) \\
\hline \multirow{4}{*}{$\begin{array}{l}\text { Low molecular } \\
\text { surfactants }\end{array}$} & Lecithin & Strong emulsifying agent; permeation enhancing effect & (Klang \& Valenta, 2011) \\
\hline & Sugar esters & $\begin{array}{l}\text { Good taste and aroma profile; capacity to form different colloidal } \\
\text { structures depending on system composition and temperature }\end{array}$ & (Rao \& McClements, 2011) \\
\hline & Ramnolipids & $\begin{array}{l}\text { Surface active properties; anionic character under appropriate } \mathrm{pH} \\
\text { conditions; good stability when heated }\end{array}$ & (Bai \& McClements, 2016) \\
\hline & Quillaja saponin & $\begin{array}{l}\text { Excellent emulsifying properties; pharmaceutical effects, including } \\
\text { antitumor and antimicrobial activities }\end{array}$ & $\begin{array}{l}\text { (Chung, Sher, Rousset, Decker, \& } \\
\text { McClements, 2017) }\end{array}$ \\
\hline
\end{tabular}

process may have several profiles such as (McClements, 2012a):

i) Burst release - quick release of most of the encapsulated compound in a short time;

ii) Sustained release - extended release of the encapsulated compound at a constant rate;

iii) Triggered release - the encapsulated compound is released in reaction to a specific environmental stimulus such as $\mathrm{pH}$, ionic strength, enzyme activity or temperature;

iv) Targeted release - the encapsulated compound is released in a specific location of the GI tract (i.e. mouth, stomach, small intestine or colon).

One of the main challenge of designing delivery systems is to have the capacity to release the nutraceutical when the system is triggered through an external factor such as $\mathrm{pH}$, moisture, enzymes, temperature or shear and at the same time, protect the nutraceutical from other factors until it is released in the site of action (McClements \& Li, 2010).

Controlled release of a desirable flavor or release of food ingredients at an appropriate time during processing or storage are some examples of applications of controlled release of encapsulated compounds (Augustin \& Sanguansri, 2012, pp. 19-48).

\subsubsection{High loading capacity}

Delivery systems must have the highest loading capacity possible and must retain effectively the encapsulated compound until it reaches a specific site of action (McClements, 2012a; McClements et al., 2009). The loading capacity $(L C)$ is a parameter that measures the capacity of a delivery system to encapsulate the bioactive compound. This parameter is determined through the mass of encapsulated material $\left(M_{\mathrm{E}}\right)$ per unit mass of carrier material $\left(M_{C}\right)$ (McClements et al., 2009):

$L C=\frac{M_{E}}{M_{C}}$
The loading capacity is dependent on the bioactive compound properties and on the encapsulation material used, such as chemical nature, molecular weight, polarity and volatility of the bioactive compound and its interactions with the food matrix (Augustin \& Hemar, 2009).

\subsubsection{Bioavailability}

Chemical instability during digestion, poor solubility in GI fluids, low intestinal absorption or first-pass metabolism are some common issues that need to be avoided to increase the nutraceuticals' bioavailability (Yao, McClements, \& Xiao, 2015). Furthermore, the bioavailability is affected by the materials used to encapsulate and their physical state, the encapsulation vehicle and the surrounding food matrix (Livney, 2015).

\subsection{Encapsulating materials}

A key step in the delivery systems' development is the selection of the encapsulating materials, once it will largely influence the encapsulation efficiency and delivery systems' stability (Faridi Esfanjani, Assadpour, \& Jafari, 2018). However, this is also a key challenge in designing food grade delivery systems for nutraceuticals due to the limited choice of materials that can be used in the formulation. As previously mentioned, most of the delivery systems developed for pharmaceutical applications use synthetic components that cannot be applied to food products due to its potential negative effects upon chronic consumption (Ting et al., 2014). So it is important to design delivery systems with bio-based ingredients, biocompatible with food products and non-toxic for human consumption (Dima, Dima, \& Iordăchescu, 2015). However, some disadvantages of the utilization of bio-based food ingredients are the difference in their properties due to the conditions and processes used for their isolation and the quality and composition of initial source (Augustin \& Sanguansri, 2012, pp. 19-48). Furthermore, encapsulating materials should have low viscosity, good 
film forming capacity, barrier and gelling properties and neutral odor and taste (Augustin \& Sanguansri, 2008). Bio-based encapsulating materials such as polysaccharides, proteins, lipids and low molecular surfactants have been widely used as encapsulating materials due to their biodegradability, biocompatibility and non-toxicity. Some examples of bio-based encapsulating materials, as well as their particular characteristics that makes them attractive candidates to be used as encapsulation materials can be found in Table 2.

Polysaccharides are the most used biopolymers in encapsulation of nutraceuticals for food applications. Polysaccharides can be applied in the formation of the delivery systems with different functions, for example as wall material in spray drying microcapsules or as structuredlayered oil-in-water $(\mathrm{O} / \mathrm{W})$ interfaces in emulsions. Hydrophobic interactions, hydrogen bonds, van der Waals forces and ionic interactions are the main interactions established between polysaccharides and nutraceuticals. Nutraceuticals' release depends mainly on the hydrolysis of the glycosidic linkages of the polysaccharides (de Vos, Faas, Spasojevic, \& Sikkema, 2010). When polysaccharides are subjected to environmental alterations such as $\mathrm{pH}$, ionic strength, temperature or solvent composition, they may undergo transition to different aggregation states and different conformations.

The incorporation of nutraceuticals within proteins has been also widely studied. This can occur using molecular complexes (e.g. $\beta$-lactoglobulin), self-assembled structures (e.g. casein micelles) or polymeric gel particles. The latter can be formed through cation-induced gelation (e.g. soy protein isolate), enzymatic (e.g. trans-glutaminase) and chemical cross-linking of gels (e.g. glutaraldehyde) (Shutava et al., 2009). The main interactions responsible for the association between nutraceuticals and protein complexes and their respective conformation are hydrophobic and electrostatic interactions, van der Waals forces, covalent bindings and hydrogen bonds (Oehlke et al., 2014).

Due to their self-assembly capabilities, lipids are also suitable for incorporation of sensitive molecules, to hide flavors and to control the release of the encapsulated molecules (McClements et al., 2009). Lipid carriers can also improve the nutraceuticals' solubilization in the GI tract, because they have the ability to stimulate the secretion of endogenous biliary-derived solubilizing components including bile salts and phospholipids (Porter, Trevaskis, \& Charman, 2007).

Surfactants, molecules composed by a hydrophilic "head" group and a lipophilic "tail" group, can spontaneously form micelles, reverse micelles, bilayers and vesicles, at high concentrations. Besides that, they can organize in different liquid crystalline structures such as lamellar, hexagonal and reversed hexagonal phases (McClements et al., 2009). Molecular structure characteristics of surfactants and the physicochemical environment where they operate determine their functional properties.

\subsection{Delivery systems design}

In order to design a delivery system suitable for a specific nutraceutical, generally, tailor made approach should be followed, depending on nutraceuticals' physicochemical properties (e.g. solubility, chemical interactions and stability) and on the final application (e.g. amount of nutraceutical required, storage conditions, type of food product and its shelf-life) (Aditya, Espinosa, \& Norton, 2017). Also, there are other important features to take into consideration, such as optimum nutraceutical concentration, release mechanism and final particle size and density (Faridi Esfanjani \& Jafari, 2016).

Delivery systems for food applications can be divided in two groups: i) lipid and surfactant-based carriers, which include emulsions, liposomes, solid lipid particles, nanostructured lipid carriers and self-dispersing lipid formulations and ii) biopolymer-based carriers (i.e. polysaccharide and protein-based carriers), which include hydrogels, protein-polysaccharide complex and polymeric micelles (Fig. 2). The main characteristics, as well as the advantages and limitations of the most important delivery systems for food applications are briefly described below.

\subsubsection{Emulsions}

Emulsions can be $\mathrm{O} / \mathrm{W}$ or water-in-oil (W/O) and liquid in liquid or liquid in solid. The droplet size and emulsion stability are dependent on the oil type and composition, the surfactant/oil ratio and co-solvents or co-solutes used. Lipids, low molecular weight surfactants, proteins and polysaccharides are the main encapsulating agents used (Livney, 2015).

There has been an increasing interest in colloidal delivery systems based on microemulsions and nanoemulsions because they can be easily produced from food-grade ingredients using processing operations such as mixing, shearing and homogenization. These two systems present many structural similarities, but there are also some important differences between them. A nanoemulsion can be defined as a thermodynamically unstable colloidal dispersion consisting of two immiscible liquids, with one of the liquids being dispersed as small spherical droplets $(r<100 \mathrm{~nm})$ in the other liquid, whereas, microemulsions refer to a thermodynamically stable colloidal dispersion consisting of small spheroid particles (comprised of oil, surfactant, and possibly co-surfactant) dispersed within an aqueous medium (McClements, 2012b).

Microemulsions tend to appear either transparent or only slightly turbid, have high solubilization capacity for lipophilic compounds, and are easy to prepare (usually easier than nanoemulsions) and scale up for commercial applications. However, there is usually a need to use high amounts of surfactants (generally much higher comparing to nanoemulsions), which could result in toxic effects (Augustin \& Hemar, 2009). In fact, microemulsions used in food industry face limitations mainly due to the restrictions in surfactants' use - there are only a limited number of food-grade surfactants available for stabilizing these systems (Chatzidaki, Mitsou, Yaghmur, Xenakis, \& Papadimitriou, 2015).

Nanoemulsions may be produced through self-emulsification methods or high pressure homogenization (Dima et al., 2015; Livney, 2015). The principal advantages of this delivery system are the production of optically transparent and kinetically stable emulsions, possibility to control the droplet size, relatively easy manufacturing and fast absorption upon oral administration. However, it must be taken into consideration that surfactant amount affects nanoemulsion droplets stabilization and size. Additionally, the use of excessive surfactants may cause toxic effects (Shin, Kim, \& Park, 2015).

Emulsions' droplet size has been shown to influence emulsions' stability. For example, the effect of droplet size on emulsions' degradation has been investigated by encapsulating lycopene-enriched tomato extract. The authors used the emulsification-evaporation method and tested different parameters of homogenization (i.e. pressure and number of cycles) and obtained nanoemulsions with droplet diameters between $96 \mathrm{~nm}$ and $286 \mathrm{~nm}$ and $\zeta$-potential between $-33 \mathrm{mV}$ and $-42 \mathrm{mV}$, which demonstrated good stability. Regarding to lycopene emulsions degradation, it was observed that droplets with smaller size have better protection against the environment and degradative factors (Ha et al., 2015). Other authors developed food grade conventional emulsions (size of $1285 \mu \mathrm{m}$ ) and nanoemulsions (size of $277 \mathrm{~nm}$ ) encapsulating vitamin $\mathrm{E}$ and they showed that nanoemulsion formulation increased the emulsions stability when compared to the conventional emulsion (Parthasarathi, Muthukumar, \& Anandharamakrishnan, 2016).

Emulsions are also able to encapsulate more than one nutraceutical in the same structure. Mucoadhesive nanoemulsions based on hyaluronic acid have been developed for co-encapsulating two polyphenols (resveratrol and curcumin). These nanoemulsions showed to preserve the polyphenols' antioxidant ability and protect them from degradation (Nasr, 2016).

\subsubsection{Liposomes}

Liposomes, sphere-shaped vesicles, are constituted by one (unilamellar) or more (multilamellar) bilayers of lipids that can incorporate 


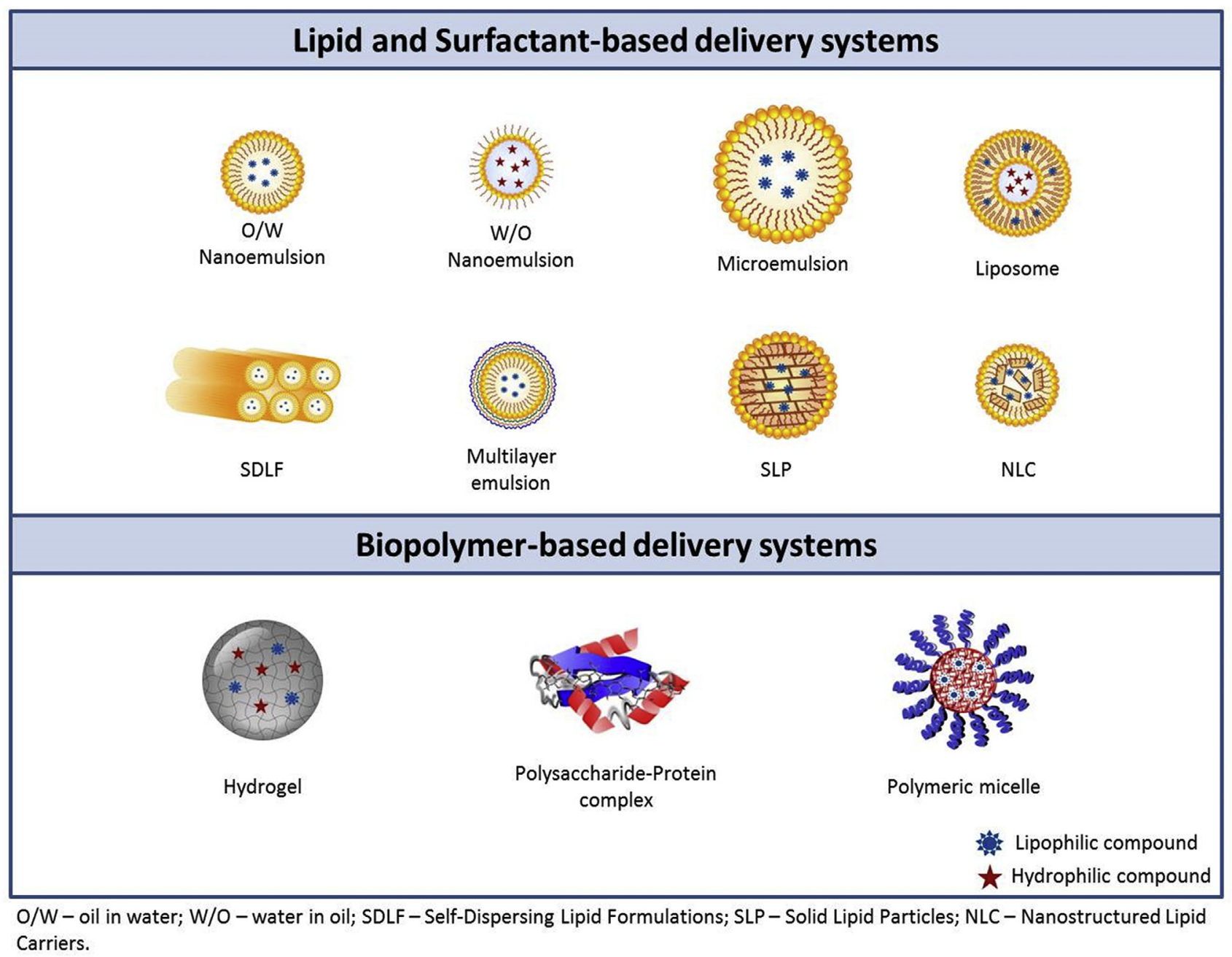

Fig. 2. Micro- and nano-delivery systems that can be used to encapsulate nutraceuticals.

hydrophilic and lipophilic nutraceuticals. Phospholipids from eggs, soy and milk are the most used materials for liposome production. Liposomes can also be produced by non-ionic surfactants, known as niosomes (Dima et al., 2015).

These systems can be produced by detergent depletion, membrane extrusion, lipid layer hydration, high pressure homogenization, ethanol injection method, sonication, reverse phase evaporation and microfluidization (Augustin \& Hemar, 2009; Dima et al., 2015). The selection of the most appropriate production method depends on type of liposomes, phospholipid properties, nutraceutical properties and the relationship between phospholipids and dispersion medium. For instance, a study has been conducted to evaluate plant sterols encapsulation in liposomes composed by soy phospholipids through different methods (Alexander, Acero Lopez, Fang, \& Corredig, 2012). It has been shown that the incorporation of plant sterols increased the liposomes' particle size and their encapsulation efficiency. Furthermore, it was concluded that the preparation methodology may interfere with liposomes' properties. Ethanol method (in which phospholipids and plant sterols are mixed with ethanol at room temperature and then heated) presented the highest encapsulation efficiency for liposomes with oil soluble sterols and better stability to storage and dilution. Other authors developed different vesicular systems to deliver baicalin, a bioactive flavonoid with various pharmacological activities. Results revealed that all vesicular systems, including liposomes, are promising vehicles for baicalin in vitro stabilization, sterilization endurance, safety and ocular pharmacokinetic (Ashraf, Nasr, Nebsen, Said, \& Sammour, 2018).

Some advantages of liposomes are biocompatibility, biodegradability, low toxicity and multi-compounds encapsulation. However, they have poor storage stability, low encapsulation efficiency, short release time and sensitivity to external factors such as light, temperature and oxygen (Dima et al., 2015; Shin et al., 2015). The low stability of liposomes can be circumvented, for example, by the addition of a coating. Salmon protein hydrolysates have been encapsulated in liposomes composed of milk fat globule membrane phospholipids coated with chitosan using different phospholipids and chitosan concentrations (Li, Paulson, \& Gill, 2015). Uncoated liposomes exhibited different sizes depending on phospholipids' concentration, and an extensive aggregation. However, they presented uniform distribution size and similar $\zeta$-potential. Regarding coated liposomes, it was observed that for higher chitosan concentrations, the particles presented similar size regardless of the phospholipids' concentration (broad size distribution), and positive values of $\zeta$-potential. Comparatively to the uncoated liposomes, coated liposomes showed higher encapsulation efficiency, stability and lower release in simulated-gastricfluids and simulated-intestinal-fluid.

\subsubsection{Solid lipid particles (SLPS)}

SLPs are composed by lipid droplets that are fully crystallized and have an organized crystalline structure with the bioactive components accommodated within the lipid matrix (Katouzian, Faridi Esfanjani, Jafari, \& Akhavan, 2017). The most used lipids are fatty acids, glycerides, paraffin, triacylglycerol and waxes. SLPs present several advantages such as controlled and targeted release, good stability, good biodegradability and biocompatibility, high loading capacity and low 
cost. However, they present low loading capacity for core materials with limited solubility in melted lipids, high water content and during storage, the core can be expelled after liquid-to-solid phase transition. They can be produced by hot or cold homogenization (Shin et al., 2015).

The incorporation of nutraceuticals into solid lipid nanoparticles (SLNs) can be divided in three different models (Fathi, Mozafari, \& Mohebbi, 2012; Ramteke, Joshi, \& Dhole, 2012):

i) Homogeneous matrix model: also known as solid solution model, the nutraceutical is present in amorphous clusters or is molecularly dispersed. It is achieved when cold homogenization method is used and when highly lipophilic compounds are incorporated in SLNs without surfactants following hot homogenization method. In the cold homogenization technique, the nutraceutical is dispersed in molecularly dispersed form in bulk of melted lipid, then the application of the mechanical force of high pressure homogenization results in the breakdown of molecular form to nanoparticles having the homogeneous matrix structure. The release process in this model is based in the dissolution mechanism;

ii) Bioactive-enriched shell: the lipid core is enclosed by a nutraceutical-enriched outer shell. Upon cooling, the lipid precipitates first, forming a solid lipid core due to phase separation. Also, the nutraceutical re-partitions into the remaining liquid phase, resulting in nutraceutical concentration in SLN outer shell. A burst release behavior is observed is this case;

iii) Bioactive-enriched core: it is obtained when the nutraceutical dissolves in the melted lipid at or close to its saturation. When the melted lipid containing the nutraceutical is cooled, supersaturation of the nutraceutical in the lipid occurs, resulting in the nutraceutical precipitation prior to lipid precipitation. Further cooling will lead to the precipitation of the lipid surrounding the nutraceutical enriched core (i.e. as a membrane). In this case, a controlled release regulated by Fick's law of diffusion is promoted.

The selection of lipids for the production of SLPs must take into account the solubility of the nutraceuticals in order to ensure good encapsulation efficiency and loading capacity, biodegradability, digestibility and lipid chemical and thermal stability (Dima et al., 2015). Also, the type of surfactant absorbed on the oil-water interface and the use of different mixtures of lipids strongly affects SLPs chemical and physical stabilities. In a recent study, the physicochemical stability of $\beta$ carotene has been improved by encapsulating this nutraceutical into SLNs containing palmitic acid and corn oil, stabilized by whey protein isolate (WPI). In this case, palmitic acid crystals covered the surface of the oil droplets and formed a solid shell to protect the encapsulated $\beta$ carotene. The addition of corn oil in combination with palmitic acid decreased the probability of $\beta$-carotene exclusion from the solid matrix to the surface of the SLNs, and the WPI was able to increase the $\beta$ carotene oxidative stability. This system showed a high stability against different harsh conditions (Mehrad, Ravanfar, Licker, Regenstein, \& Abbaspourrad, 2018).

\subsubsection{Nanostructured lipid carriers (NLCs)}

Some authors consider NLCs derived from $\mathrm{O} / \mathrm{W}$ nanoemulsions (Tamjidi, Shahedi, Varshosaz, \& Nasirpour, 2013) and others a modified version of SLNs (Shin et al., 2015). NLCs are nanostructures where the lipidic phase is composed by liquid-lipid and solid-lipid mixtures at room temperature. This system was developed essentially to overcome the limitations of SLNs; indeed, the mainly advantages of this system are higher encapsulation efficiency, loading capacity, physical stability, control release, low toxicity, biodegradability and bioavailability (Shin et al., 2015; Tamjidi et al., 2013). Due to these attractive characteristics, in the recent years there has been as increasing interest in NLCs for encapsulation of nutraceuticals. Rutin-loaded NLCs have been produced using cacao butter, oleic acid and Tween 80 through high shear rate homogenization and their incorporation in food models was evaluated. The authors observed that particles' size increased between $77 \mathrm{~nm}$ and $96 \mathrm{~nm}$ with the increase of rutin/lipid (R/L) ratios; however, during the storage time all different ratios tested maintained their physical stability. Furthermore, the R/L ratios of $5 \%$ and $10 \%$ presented good stability during the storage period and few amounts of rutin were released. When incorporated in food models, NCLs have shown to be stable during storage and processing time and they did not affect the appearance of the food models (Babazadeh, Ghanbarzadeh, \& Hamishehkar, 2016). Other authors encapsulated different types of terpenes in NLCs produced by ultrasound homogenization using modified beeswax as solid lipid and caprylic triglyceride as liquid lipid. They obtained NLCs with particle sizes around $97 \mathrm{~nm}$ and $106 \mathrm{~nm}$ with $\zeta$-potential values above $-30 \mathrm{mV}$, which demonstrate a good physical stability for the different types of terpenes. Relatively to the release study, overall, it was observed a burst release in the first hours and then a gradual release. Furthermore, it has been observed that terpenes with lower lipophilicity tend to remain linked to lipid nanoparticles for longer time than higher lipophilic terpenes (Lasoń, Sikora, Ogonowski, Tabaszewska, \& Skoczylas, 2016).

To choose a suitable lipid blend, it is necessary to take into account the following factors: i) solubility of the nutraceuticals in the lipid matrices; ii) the solid and liquid lipids need to be miscible at specific concentrations, but their molecules should be spatially incompatible together; iii) the lipid phase should be stable to chemical degradation; and iv) the lipid should have low toxicity and not produce toxic residues during the NLCs preparation. Medium Chain Triglycerides (MCT), oleic acid and natural edible oils are the most used liquid oils whereas the most used solid lipids are glycerides, fatty acids and waxes (Dima et al., 2015). Similar to SLNs, NLCs could be prepared through cold or hot homogenization. NLCs can be divided into three types: i) imperfect type, in which NLCs are produced with solid and liquid lipids chemically very different from each other, resulting in systems with imperfections or holes where the nutraceutical are accommodated in molecular form; ii) amorphous type, in which the lipid core solidifies in amorphous state instead of crystalline state; and iii) multiple type, where the solid lipid matrix has small liquid nano-compartments of oil where the nutraceuticals are dissolved, increasing the loading capacity and the possibility of controlled release (Tamjidi et al., 2013).

\subsubsection{Self-dispersing lipid formulations (SDLF)}

SDLFs are isotropic oil solutions or isotropic mixtures of oils, surfactants, co-surfactants and co-solvents. However, these systems are not emulsions, they only transform in emulsions when in contact with water or biological fluids (Dima et al., 2015; Shin et al., 2015). The principal advantage of this system is the improvement of nutraceuticals' solubility in the GI tract which enhance their adsorption. SDLF bioavailability depends on the digestion of lipids, lipophilicity of nutraceuticals, type of lipids and on the mean droplet diameter (Pathak \& Raghuvanshi, 2015). Monoglycerides are the most used oils and can present different self-assembly structures when in contact with water. They can be dispersed in vesicles or liposomes structures for lamellar phases, or in cubosomes (bicontinuous cubic particles) and hexosomes (bicontinuous hexagonal particles) for cubic or hexagonal phases, respectively. Their main applications in food products are the controlled release of aroma, creation of flavors compounds through Maillard reaction and structure food products (Augustin \& Hemar, 2009). One disadvantage of these systems is the utilization of large amounts of surfactants and their toxicological risks; however, there are not many studies on this yet (Augustin \& Hemar, 2009; Dima et al., 2015).

Quercetin has been encapsulated in self-emulsifying drug delivery system (SEDDS) composed of Capmul MCM as the oil phase, Tween 20 as surfactant and ethanol as co-surfactant and its stability and antioxidant capacity was evaluated. Emulsions with globule size of $77 \mathrm{~nm}$, polydispersity of 0.24 and $\zeta$-potential value of $-19.5 \mathrm{mV}$ have been obtained. The system did not show separation of phases or turbidity 
until after 6 months and showed robustness and stability for different dilutions and different values of $\mathrm{pH}$. Furthermore, SEDDS were stable when subjected to freeze/thaw and accelerated stability tests. The developed SEDDS with quercetin showed similar antioxidant activity when compared to free quercetin, showing that quercetin's properties remained even in the presence of SEDDS components (Jain, Jain, Pohekar, \& Thanki, 2013).

\subsubsection{Biopolymer-based delivery systems}

Biopolymers (e.g. polysaccharides and proteins) can be used to create a wide range of delivery systems for nutraceuticals, either individually or combined.

Polymeric particles can be produced with common food biopolymers through self-assembly and can be used to encapsulate both hydrophilic and hydrophobic nutraceuticals. Their main advantages are high loading efficiency, high stability, biocompatibility, biodegradability and controlled release (McClements et al., 2009; Shin et al., 2015).

Polymeric micelles are formed above the critical micelle concentration of amphiphilic biopolymers which have both hydrophobic and hydrophilic functional groups. These structures are constituted by a hydrophilic region (shell) and a hydrophobic part (core). Their main advantages are low toxicity, high loading capacity and improved water solubility. The most used polymeric micelles for encapsulation of nutraceuticals are casein micelles (Shin et al., 2015). As an example, docosahaxaenoic acid (DHA) has been bonded to casein and then, entrapped within re-assembled casein micelles. Casein micelles with a size of $50 \mathrm{~nm}$ and $60 \mathrm{~nm}$ were obtained, which were not affected by the thermal treatment. Also, this system showed a remarkable protective effect against DHA oxidation, good colloidal stability and bioactive conservation throughout shelf life (Zimet, Rosenberg, \& Livney, 2011).

Biopolymer-derived hydrogels have also recently captured considerable attention as promising delivery systems for nutraceuticals (Abaee, Mohammadian, \& Jafari, 2017). Hydrogels can be defined as networks of hydrophilic polymers formed by covalent and hydrogen bonds, van der Waals interactions or physical entanglements. They are able to absorb large amounts of water while maintaining their internal network structure. These systems also have a high loading capacity for nutraceuticals and targeted release ability, being suitable to be used as delivery systems. In particular, nanohydrogels combine hydrogels' properties (such as hydrophilicity and high water content) with those of nanotechnology (i.e. very small sizes), exhibiting a unique potential as nanoscale delivery systems (Mokhtari, Jafari, \& Assadpour, 2017). Also, nanohydrogels can be taken up by cells and may more easily access some areas of body that are impossible for hydrogels. They can be produced by physical self-assembly or chemical cross-linking of amphiphilic or hydrophilic polymers. The principal advantages of nanohydrogels are their high stability, high loading capacity, response capacity to environmental stimuli and high water content (McClements et al., 2009; Shin et al., 2015). This type of system has also gained considerable interest as a delivery system. Recently, curcumin and caffeine have been incorporated in lactoferrin-glycomacropeptide nanohydrogels and their ability to encapsulate and release both lipophilic and hydrophilic compounds was evaluated. The authors obtained nanohydrogels with sizes of $170 \mathrm{~nm}, 112 \mathrm{~nm}$ and $126 \mathrm{~nm}$ for empty nanohydrogels, nanohydrogels with curcumin and nanohydrogels with caffeine, respectively. It was observed that nanohydrogels are capable to encapsulate both lipophilic and hydrophilic compounds, with encapsulation efficiencies of $95 \%$ and $90 \%$ for curcumin and caffeine, respectively. The release of these bioactive compounds was also evaluated at $\mathrm{pH} 2$ and $\mathrm{pH} 7$, and it was observed that the medium $\mathrm{pH}$ has a significant effect on their release (Bourbon, Cerqueira, \& Vicente, 2016).

Polysaccharide-protein complexes consist in the combination of two or more biopolymers - polysaccharides and proteins - and can be defined as coacervates or precipitates. Coacervates are open structures rich in water that can be formed when biopolymers have opposite net charges and the complex's net charge does not oppose the higher-order association. Precipitates are also formed by biopolymers with opposite net charges, but in this case, are promoted when biopolymers interactions are strong enough to release water and counter-ions.

One disadvantage of coacervates is their instability and tendency to coalesce when subjected to differences of $\mathrm{pH}$ or ionic strength. The stabilization of these structures can be promoted by adding glutaraldehyde or multivalent ions, heating treatment or enzymatic treatment (McClements et al., 2009). It has been reported that nanocomplexes of $\beta$-lactoglobulin and pectin encapsulating DHA present good stability and particle size (between $110 \mathrm{~nm}$ and $272 \mathrm{~nm}$ ), resulting in clear suspensions appropriate for application in clear beverages. Moreover, these complexes showed to be able to protect DHA from degradation (Zimet \& Livney, 2009).

\subsubsection{Nanolaminated systems}

Multilayers of different materials can be assembled on top of preexistent delivery systems (e.g. emulsions, liposomes, polymeric particles), forming nanolaminated systems. Layer-by-layer (LbL) is the most used method for producing nanolaminated systems and consists in the adsorption of charged layers of polyelectrolytes on top of the core material. The main advantages of LbL technique are the simplicity of the process and equipment, capability of application in several sizes and irregular shapes, with control of the size, composition, porosity, stability, surface functionality and colloidal stability (McClements \& Li, 2010). As the different layers are connected together through electrostatic attraction, it is possible that these layers dissociate with the change in $\mathrm{pH}$ or ionic strength. To ensure the connection between layers, LbL systems may be subjected to heating, enzymatic and chemical treatments (McClements et al., 2009).

Multilayer emulsions can be produced by coating the nutraceuticalscontaining oil droplets with one or more charged layers. Their production requires high control of $\mathrm{pH}$, ionic strength, type and concentration of polyelectrolyte, emulsifiers and the sequence of compound-adding. Some advantages of multilayer emulsions are the capability to incorporate lipophilic and hydrophilic compounds, improvement of the solubility of lipophilic components, bioadhesive properties, permeability through biological membranes, better physical and chemical stability to environmental stresses, capacity to trigger the release of the nutraceutical and control the release rate (Dima et al., 2015; McClements et al., 2009). In fact, the incorporation of lactoferrin in bi-layer nanoemulsions stabilized by WPI has been shown to improve their environmental stability namely, $\mathrm{pH}$ changes and salt addition (Teo, Lee, \& Goh, 2017).

Multilayer nanocapsules can be produced using the LbL technique followed by dissolution of a colloidal template in acid or solvent and several centrifugation-washing cycles (Pinheiro et al., 2015). These systems have the ability to protect and release the nutraceuticals in the specific site of action (e.g. small intestine, colon). Recently, folic acid has been incorporated in nanolaminates developed by the LbL deposition of alginate and chitosan, and it was observed that folic acid entrapped in nanolaminates was more stable under ultraviolet light exposure than non-encapsulated folic acid. Also, the release profiles of folic acid were affected by $\mathrm{pH}$ conditions, showing a greater release at $\mathrm{pH} 7$ compared to $\mathrm{pH} 3$, suggesting that the developed nanolaminates might exhibit a controlled release in the GI tract (i.e. scarce release at the stomach and a burst release in the small intestine) (Acevedo-Fani, Soliva-Fortuny, \& Martín-Belloso, 2018). Other authors developed a multilayer coating based on $\kappa$-carrageenan and quercetin-loaded lecithin/chitosan nanoparticles by LbL technique (Souza et al., 2018). This multilayer coating showed to have good antioxidant capacity and did not present cytotoxicity. 

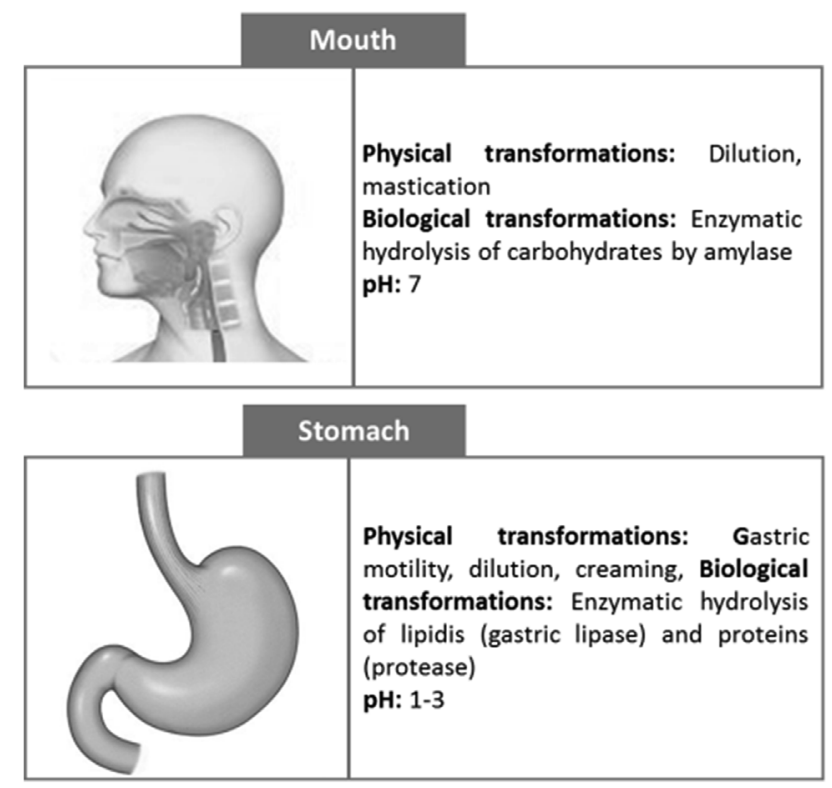

Small intestine
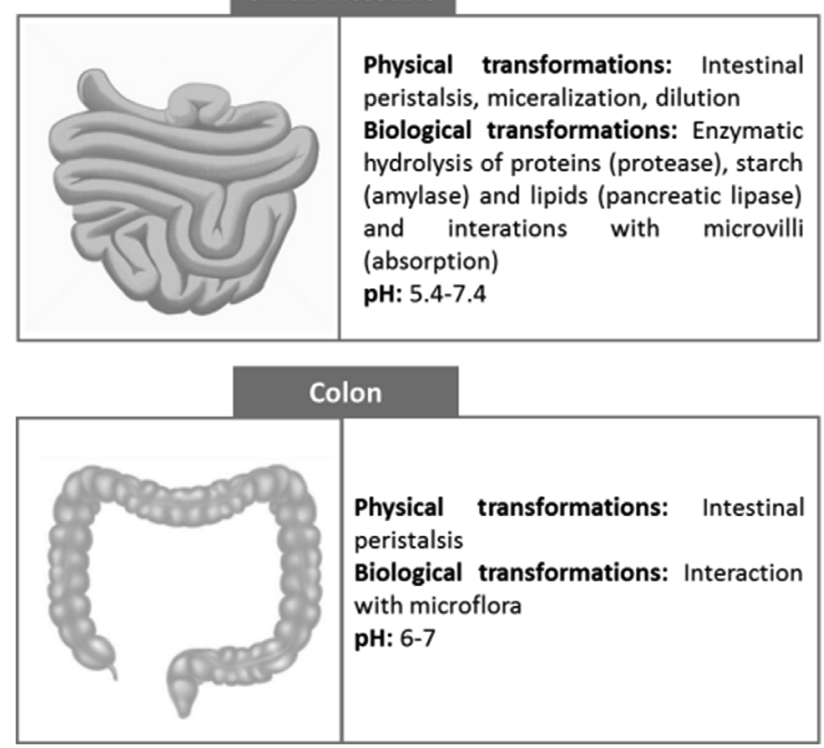

Fig. 3. Physiological and physicochemical changes of delivery systems that can occur within the human gastrointestinal tract (adapted from (Pinheiro, Gonçalves, Madalena, \& Vicente, 2017)).

\section{Gastrointestinal fate of delivery systems/nutraceuticals}

Ingested delivery systems undergo a series of complex physicochemical and physiological processes as they pass through the different regions of the human GI tract, before the release of nutraceuticals (Fig. 3).

\subsection{Changes in delivery systems' properties in the GI tract}

Mouth: Right after ingestion, delivery systems can experience changes in their composition, structure and properties due to the complex processes occurring within human mouth. In the oral phase, delivery systems are mixed with saliva, being diluted and submitted to a complex forces/flow profile. Also, there may be changes in their $\mathrm{pH}$, ionic strength and temperature; they can interact with the surface of the tongue and mouth; they can be physically broken down into smaller pieces by chewing and, depending on their composition, they may be hydrolyzed by the digestive enzymes which, is the case of starch that is hydrolyzed by amylase (McClements, 2014a). Therefore, the characteristics of the delivery systems after exposure to oral conditions can be very different from the initial characteristics. For example, it has been shown that nanoemulsions with different lipid phase compositions significantly increased their particle size after passing through the mouth phase (Zhang, Zhang, Kumosani et al., 2016). After this phase, delivery systems are transformed in a form suitable for swallowing (bolus) and pass down the esophagus, entering the stomach (Tso, 2000).

Stomach: Within the stomach, the delivery systems are submitted to harsh physicochemical and physiological conditions that may further change its composition and structure. In fact, the high acidity (i.e. $\mathrm{pH}$ between 1 and 3) may cause the degradation of some of the components within the colloidal delivery systems. Also, the alterations in $\mathrm{pH}$ and ionic strength (that present high values around $100 \mathrm{mM}$, being $\mathrm{Na}^{+}$, $\mathrm{K}^{+}, \mathrm{Ca}^{2+}$ and $\mathrm{Cl}^{-}$the major species) may lead to changes in the electrical characteristics of ionizable groups, and therefore in the electrostatic interactions of the system, that could have direct consequences in the integrity, permeability or aggregation of the colloidal delivery systems. Moreover, the gastric enzymes may hydrolyze some components of the delivery systems (i.e. gastric lipases initiate lipid digestion, whereas gastric proteases begin protein digestion) and surface-active substances (e.g. phospholipids and proteins) present in the gastric juices may adsorb to the surfaces of the delivery systems, altering their surface characteristics. Delivery systems are also submitted to complex flow/force patterns (i.e. gastric motility) that mix them with the gastric juices, break down any remaining large fragments, as well as transfer the digested material into the small intestine (McClements, Decker, \& Park, 2008). The type and composition of the delivery system will influence the degree and nature of the changes that occur within the stomach. For instance, it has been shown that protein-stabilized nanoemulsions are prone to coalescence phenomena at some extent within the stomach. However, higher instability was observed for multilayer nanoemulsions (stabilized by a protein and a polysaccharide) that exhibited a large increase in the particle size (indicative of an extensive droplet aggregation) and a high evidence of creaming (Pinheiro, Coimbra, \& Vicente, 2016). Other authors also confirmed that the delivery systems behavior under gastric conditions is highly influenced by their composition: surfactant-stabilized emulsions have shown to be stable within the gastric environment, whereas the protein-stabilized emulsions exhibited extensive flocculation, leading to creaming (van Aken, Bomhof, Zoet, Verbeek, \& Oosterveld, 2011).

Small intestine: The partially digested material that leaves the stomach and enters the small intestine is usually referred to as "chyme". The chyme is mixed with small intestine fluids: i) sodium bicarbonate is secreted to increase $\mathrm{pH}$ from highly acidic to closer to neutral (typically within the range of $\mathrm{pH} 5.4$ to 7.4 ), in order to favor the pancreatic enzymes activity; ii) bile salts and phospholipids (surface active compounds) are secreted to facilitate emulsification of the lipids by adsorbing to the droplet surfaces; iii) pancreatic enzymes are secreted to further digest any macronutrients remaining within the chyme (proteins are converted to peptides/amino acids by proteases, triacylglycerols are converted to monoacylglycerols/free fatty acids by lipases, and starches are converted into oligosaccharides/glucose by amylases), and iv) co-lipase is secreted in order to achieve the optimum lipase activity (McClements et al., 2008). In addition to sodium bicarbonate, other salts are secreted into the small intestine, being the ionic strength around $140 \mathrm{mM}$ (Lindahl, Ungell, Knutson, \& Lennernäs, 1997), which influences the magnitude and range of any electrostatic interactions occurring in the delivery system. Small intestine is considered the main site for nutraceuticals' absorption. In particular, lipophilic compounds' solubilization, transport and absorption is enhanced by the mixed micelles' formation that transport the solubilized components through the mucus layer until they reach the surfaces of the enterocytes where they are absorbed (van Aken, 2010). The behavior of the delivery systems 
during intestinal digestion is dependent on their composition. It has been shown that the emulsifier charge used to produce nanoemulsions has a significant effect on their size, charge and microstructure during digestion (in particular at duodenum, jejunum and ileum stages), consequently, influencing the free fatty acids release and nutraceuticals' bioavailability (Pinheiro et al., 2013).

Colon: Only a fraction of the components reaches the colon, once the majority of the ingested food is broken down and absorbed in the upper GI tract (i.e. stomach and small intestine). Therefore, only delivery systems composed of indigestible components (e.g. indigestible oil or dietary fibers) are expected to reach the colon without being absorbed (McClements, 2014a). Colon has a near neutral pH and contains a wide range of viable bacteria. In fact, as many as 400 species of bacteria can be found there, being the predominant species Bacteroides, Bifidobacterium and Eubacterium (Sinha \& Kumria, 2003). These bacteria are able of breaking down various food components that are not digested in the upper gut (Basit, 2005). It is clear that the colon complex microbial ecosystem has a significant impact in nutraceuticals' bioavailability. For example, it has been demonstrated that the mutual relationship between microbiota and phenolic compounds increases the phenolic compounds' bioavailability and provides increased health benefits (Ozdal et al., 2016). The main fermentation products (produced by the digestive enzymes released from colonic bacteria) of carbohydrates and proteins in the colon are short chain fatty acids that may be beneficial to human health (Sinha \& Kumria, 2003). In addition, colon bacteria are able to metabolize protein-based nutraceuticals (Tozaki et al., 1997).

\subsection{Mechanisms of nutraceuticals' release}

Different physicochemical mechanisms can be involved in the release of nutraceuticals from the matrix surrounding it and, depending on the system and environmental conditions, a different mechanism may prevail. The release can be classified into the following types:

i) Diffusion-controlled release: It is the most important mechanism used to control the nutraceuticals' release. The release rate of the nutraceutical depends on the size, shape, structure and composition of the delivery system, and is driven by the concentration gradient between the interior of the particle and the surrounding medium (Crank, 1975).

ii) Swelling-controlled release: When immersed in liquid media, hydrophilic polymers gradually start to hydrate, causing relaxation of the polymer chain with consequent volume expansion - swelling - and the nutraceutical can diffuse out (Berens \& Hopfenberg, 1978). In this case, the nutraceuticals' release rate is controlled by the water diffusion rate and by the polymers' chain relaxation rate (Peppas, Bures, Leobandung, \& Ichikawa, 2000).

iii) Erosion-controlled release: The nutraceutical is released from the matrix by erosion of its outer layer, for example by physical, chemical or enzymatic degradation. The release rate depends on the erosion rate, which is influenced by the composition and structure of the outer layers of the matrix and by the magnitude and duration of the factor that causes erosion (e.g. shear force, $\mathrm{pH}$ and enzymes) (McClements, 2012a).

iv) Fragmentation-controlled release: The nutraceutical is released when the matrix material is physically, chemically or enzymatically disrupted. In this case, the release rate will depend on the stress applied to cause the fracture, as well as on the size and shape of the fragments formed (McClements, 2012a).

v) Dissolution-controlled release: The nutraceutical is released from the delivery system as a result of a dissolution process when the delivery system is exposed to specific environmental conditions. The release rate is governed by the dissolution rate, which depends on the composition and structure of the delivery system, as well as on the magnitude and duration of the environmental factor that causes dissolution (e.g. dilution, solvent type, $\mathrm{pH}$, ionic strength or temperature) (McClements, 2014b).

vi) Stimuli-controlled release: The release of nutraceuticals is activated by internal or external stimuli such as temperature (Fundueanu, Constantin, Ascenzi, \& Simionescu, 2010), pH (Fuciños et al., 2014) or ionic strength (Bawa, Pillay, Choonara, \& Toit, 2009).

\subsection{Absorption of delivery systems/nutraceuticals}

As previously mentioned, small intestine is considered the main site for delivery systems/nutraceuticals absorption. The intestinal wall is a complex and active structure that guarantees intestinal equilibrium by regulating nutrient absorption, interactions between intestinal microflora and immune system (Davitt \& Lavelle, 2015). Before reaching the endothelia cells, nutraceuticals or delivery systems must first diffuse through the thick intestinal mucus layer. This mucus layer is synthesized by goblet cells and it is composed by a combination of glycoproteins and lipids (Boegh \& Nielsen, 2015). Different properties influence the ability to pass through the mucus layer such as size, charge and viscosity (Lai, Wang, \& Hanes, 2009). Permeability of large molecules or mucoadhesive lipophilic compounds may be reduced when interacting with mucus. Nutraceuticals may have limited capacity to permeate the gut endothelia. However, lipophilic compounds, such as resveratrol and kaempferol, mixed with phospholipids, free fatty acids and bile salts may pass through the intestinal mucus (Gleeson, Ryan, \& Brayden, 2016). On the other hand, nutraceuticals entrapped in nanostructures coated with polymers such as polyethylene glycol (PEG), chitosan, lectin and gelatin, can pass through or adhere to the mucus layer allowing nutraceuticals' uptake (Mansuri, Kesharwani, Jain, Tekade, \& Jain, 2016). Nano-delivery systems with negative surface charges can be repelled by the mucus layer, decreasing the residence time in the epithelial cells, thus lowering cellular uptake (Hariharan et al., 2006). It has been reported that positively charged poly(lactic-coglycolic acid) (PLGA) nanoparticles loaded with estradiol had an increased cellular uptake compared to negatively and neutrally charged nanoparticles (Hariharan et al., 2006).

After diffusion through the mucus, two major routes may be considered when nutraceuticals or nano-delivery systems cross the intestinal epithelium barrier, namely paracellular (through tight junctions) and transcellular (including M-cell-mediated transport) routes.

\subsubsection{Paracellular route}

Paracellular route is responsible for passive transport of molecules through intercellular spaces between intestinal epithelial cells (Daugherty \& Mrsny, 1999). Intestinal permeability is regulated by epithelial tight junctions (TJ), which are a complex structure composed by different proteins such as occludin and claudin, positioned at the intercellular space (Lerner \& Matthias, 2015). They are necessary to maintain intercellular adhesion, to regulate paracellular transport, to limit the access of microbes to host tissues, and to mediate the molecules' passage from the lumen to the lamina propria (Bischoff et al., 2014). TJ allow the passage of small hydrophilic and polar molecules (e.g. water, ions, sugars, peptides and amino acids) with a molecular weight lower than 500 Da (Maher, Mrsny, \& Brayden, 2016). It has been reported that certain compounds increase TJ functionality (such as polyphenols) while other compounds (such as caprylic fatty acid) reduce $\mathrm{TJ}$ barrier function, enhancing uptake of other small molecules (Bohn et al., 2015). Paracellular transport possibly reduces intracellular metabolism, which is relevant for nutraceuticals. However, a previous study has demonstrated that polyphenols, including caffeic acid, quercetin, chrysin, gallic acid, resveratrol and rutin are poorly transported via the passive diffusion pathway in both Caco-2 cells and parallel artificial membrane permeability assay (Rastogi \& Jana, 2014). On the other hand, the passage of nano-delivery systems across the intestinal epithelium using the paracellular route is very limited because $\mathrm{TJ}$ 
cannot open more than $20 \mathrm{~nm}$; thus, this space is constricted for a majority of nano-delivery systems (Yu, Yang, Zhu, Guo, \& Gan, 2016). Nano-delivery systems with size lower than $20 \mathrm{~nm}$ may reversibly disturb $\mathrm{TJ}$ and release the nutraceuticals to blood. Afterwards, $\mathrm{TJ}$ function returns to their regular position (Li, Jiang, $\mathrm{Xu}, \& \mathrm{Gu}, 2015$ ). Moreover, due to the negative charge present at the membrane surface, it has been shown that positive nanoparticles can be easily transported by the paracellular route (Park, Chang, \& Hoboken, 2011; Yu et al., 2013). Nano-delivery systems containing the cationic compound chitosan have thus the ability to open $\mathrm{TJ}$, enhancing paracellular transport (Chen et al., 2013). For instance, poly (glycolic acid) (PGA) and chitosan-based nanoparticles as carriers of tea catechins have been reported to cross the intestinal barrier via paracellular transport mechanism (Tang et al., 2013).

\subsubsection{Transcellular route}

The transcellular route consists on the transport (passive or active) of molecules through cells by endocytosis. Most nutraceuticals are known for being absorbed by simple transcellular route - passive transport - without requiring receptors or carriers (Renukuntla, Vadlapudi, Patel, Boddu, \& Mitra, 2013). Transcellular passive diffusion has been proposed for carotenoids and apolar polyphenol aglycones (Bohn et al., 2015). For example, SLNs loaded with curcumin permeate through Caco-2 cells using the passive diffusion mechanism (Guri, Gülseren, \& Corredig, 2013).

In contrast, there is another mechanism, which involves expenditure of metabolic energy - active transport. Some biomolecules (e.g. polar or charged molecules) bind to a specific naturally-occurring membraneprotein transporter (carrier-mediated transport) or receptor (receptormediated transport), located in the apical cell membrane, and they are transported within the intestinal cell against concentration gradient (Yun, Cho, \& Park, 2013), otherwise they could not diffuse across the cell membrane (Park et al 2011). These membrane carriers and receptors are important to the uptake of many nutraceuticals. For instance, vitamin C, fatty acids and some peptides are carried on by sodium vitamin $\mathrm{C}$ co-transporter, fatty acid-binding proteins, and proton coupled peptide transporter, respectively (Gleeson et al., 2016). Also, many polyphenols present the capacity of inhibiting or stimulating membrane transporters, which depend on the polyphenol form, concentration, exposure time, etc. (Bohn et al., 2015).

Molecules that bind to specific cell membrane receptors or carriers at the apical cell membrane are internalized in the cell by endocytic processes, which include phagocytosis or pinocytosis (Kettiger, Schipanski, Wick, \& Huwyler, 2013). Phagocytosis is mainly restricted to the M-cells of the Peyer's patch (M-cells mediated transport) (des Rieux et al., 2007). These cells, specialized in antigen sampling, offer an alleged route to nano-delivery systems (Yun et al., 2013). Once M-cells represent less than $1 \%$ of total intestine area, the transport via these cells is very difficult to occur (Acosta, 2009). Nano-delivery systems could also be internalized by pinocytosis, a mechanism that allows internalize extracellular molecules through the binding to complementary cell surface receptors (e.g. lactoferrin, lectins and $\alpha 5 \beta 1$ integrin) (Plapied, Duhem, des Rieux, \& Préat, 2011). Some nano-delivery systems have been using a receptor-specific ligand on their surface to achieve or enhance specific intracellular delivery of nutraceuticals, in both enterocytes and M-cells (Zhang \& Wu, 2014). For instance, lactoferrin nanoparticles containing gambogic acid were transported through cell membrane due to the presence of lactoferrin receptor (Zhang et al., 2013). It is also important to mention that some nutraceuticals are transported back into the lumen of the GI tract by efflux transporters present in the phospholipid bilayers, after absorption by epithelium cells. Thus, the uptake of certain nutraceuticals into the systemic circulation could be limited (Misaka, Müller, \& Fromm, 2013).

Therefore, the understanding of the different transport mechanisms across GI tract is essential for the development of effective nutraceuticals' nano-delivery systems.

\subsection{Degradation/metabolism/retention of nutraceuticals in the body}

Nutraceuticals may be metabolized by different enzymes or undergo chemical degradation (e.g. hydrolysis and oxidation) during their passage through the GI tract before reaching epithelium cells. For example, bioactive proteins or peptides may be hydrolyzed in the stomach due to the presence of proteases and low $\mathrm{pH}$, and fatty acids are targeted by pancreatic lipases (Davitt \& Lavelle, 2015). In contrast, metabolism can be useful for some nutraceuticals to be absorbed into the systemic circulation. It has been reported that quercetin glycoside (quercetin-4'-Oglucoside) is absorbed in the small intestinal through a sodium-glucose transporter and hydrolyzed by intracellular $\beta$-glucosidases, after which it passively diffuses across the basolateral membrane (Lotito, Zhang, Yang, Crozier, \& Frei, 2011). However, nutraceuticals' bioavailability and activity may be limited due to metabolism within the GI tract. An example is curcumin that undergoes a series of metabolic reactions due to $\mathrm{pH}$ variations in the GI tract resulting in a variety of metabolites with different properties (McClements, Zou et al., 2015). Also, the metabolic activity of intestinal microbiota on nutraceuticals can change host exposure to these compounds and their potential health effects. Moreover, colon constitutes a metabolism active site rather than a simple excretion route (Laparra \& Sanz, 2010). Following absorption, nutraceuticals may be distributed via portal blood or via lymphatic system to liver and spleen (Griffin et al., 2016). For example, resveratrol is rapidly metabolized in the liver and intestine, resulting in a low oral bioavailability. Thus, delivery systems may be a good approach to protect and stabilize nutraceuticals from chemical transformations and metabolism until they are released near the site of absorption. For instance, delivery systems at nano-scale are generally more abundant in kidney, liver and spleen as compared with larger-sized systems, which stay inside the GI tract (Piperigkou et al., 2016). When nano-delivery systems pass the GI barrier and go to the systemic blood circulation, they are distributed throughout the body, accessing cells and organelles that internalize and retain these structures. Some of the potential consequences of cellular interactions are cytotoxicity, inflammation, oxidative stress, and genotoxic effects (Rossi et al., 2014). The accumulation of delivery systems composed of proteins and polysaccharides would be improbable because they are digested in the stomach, small intestine or colon. However, transformations and retention of delivery systems in the body are strictly related to their physicochemical properties; thus, a case-bycase approach for evaluating efficacy and safety is required.

\section{Oral bioavailability of nutraceuticals}

Bioavailability is dependent on digestion, release from food matrix, absorption by intestinal cells, and transport to the target body cell. Only the nutraceutical fraction that reaches the blood in an active form is distributed to the organs and tissues where it can exert its beneficial health effects.

\subsection{Factors influencing oral bioavailability}

In order to develop a successful strategy to improve the bioavailability of a nutraceutical, the main factors limiting its bioavailability have to be established. The following equation summarizes the major factors influencing the bioavailability $(B A)$ of a nutraceutical (McClements, Li et al., 2015):

$B A=B^{*} \times A^{*} \times T^{*}$

Where $B^{*}$ is the fraction of the nutraceutical that is bioaccessible (i.e. potentially available for absorption (Etcheverry, Grusak, \& Fleige, 2012)), $A^{*}$ is the fraction of the bioaccessible nutraceutical that is absorbed through the layer of epithelial cells, and $T^{*}$ is the fraction of the absorbed nutraceutical that reaches the site of action in a metabolically 
active form. In vivo nutraceuticals' bioavailability is typically determined by measuring the area under the curve (AUC) (concentration $v s$. time) in the blood following the ingestion of a known amount of nutraceutical, compared to the AUC in the blood following intravenous injection of the same amount of nutraceutical (Joye, Davidov-Pardo, \& McClements, 2014).

The use of classification schemes to characterize nutraceuticals in terms of the factors that limit their bioavailability may lead to a faster identification of the appropriate delivery strategies to increase their bioavailability. According to the "Biopharmaceutical Classification System" (BCS) there are four classes of nutraceuticals: class I - high solubility and permeability compounds; class II - low solubility and high permeability compounds; class III-high solubility and low permeability compounds; and class IV—low solubility and permeability compounds (Amidon, Lennernäs, Shah, \& Crison, 1995). Other scheme has been recently developed to characterize the major factors limiting the nutraceuticals bioavailability in food - Nutraceutical Bioavailability Classification Scheme (NuBACS) (McClements, Li et al., 2015). According to this scheme, the bioavailability limiting factors can be divided into the following categories: bioaccessibility $\left(B^{*}\right)$; absorption $\left(A^{*}\right)$; and transformation $\left(T^{*}\right)$, being each one of these categories divided in sub-categories in order to describe the precise mechanism involved. The sub-categories for $B^{*}$ are: poor liberation from the food matrix $(L)$, poor solubility in the GI fluids $(S)$ and interactions that promote insolubility (I); for $A^{*}$, the sub-categories are: poor transport through the mucus layer $(M L)$, through the $T J$, or through the bilayer membrane $(B P)$, the inhibition of active transporters $(A T)$, or the presence of efflux transporters (ET) in the epithelium cells; and for $T^{*}$, the sub-categories are: changes in bioactivity is due to chemical degradation $(C)$ or metabolism $(M)$. Also, each category is flowed by the symbol "(+)" when it is a non-limiting factor and " $(-)$ " when it is a limiting factor (McClements et al., 2016)). Table 3 presents a brief overview of the application of both BCS and NuBACS systems to various nutraceuticals.

Also, there are several other physicochemical/physiological processes that may contribute to nutraceuticals low bioavailability such as

Table 3

Selected examples of nutraceuticals' classification according to BCS (Shin et al., 2015) and NuBACS (McClements, Li \& Xiao, 2015) systems.

\begin{tabular}{|c|c|c|c|}
\hline \multirow[t]{2}{*}{ Nutraceutical } & \multirow[t]{2}{*}{ Potential Health Benefits } & \multicolumn{2}{|l|}{ Class } \\
\hline & & BCS & NuBACS \\
\hline$\beta$-carotene & $\begin{array}{l}\text { Antioxidant } \\
\text { Anti-aging } \\
\text { Preventing heart disease }\end{array}$ & IV & $\begin{array}{l}\mathrm{B}^{*}(-)_{\mathrm{L}, \mathrm{I}, \mathrm{S}} \\
\mathrm{A}^{*}(-)_{\mathrm{TJ}, \mathrm{AT}, \mathrm{ET}} \\
\mathrm{T}^{*}(-)_{\mathrm{C}}\end{array}$ \\
\hline Curcumin & $\begin{array}{l}\text { Anti-inflammatory } \\
\text { Antioxidant } \\
\text { Anticancer }\end{array}$ & IV & $\begin{array}{l}\mathrm{B}^{*}(-)_{\mathrm{L}, \mathrm{S}} \\
\mathrm{A}^{*}(-)_{\mathrm{TJ}, \mathrm{AT}, \mathrm{ET}} \\
\mathrm{T}^{*}(-)_{\mathrm{M}, \mathrm{C}}\end{array}$ \\
\hline Quercetin & $\begin{array}{l}\text { Lower blood lipid } \\
\text { Anti-inflammatory } \\
\text { Antioxidant }\end{array}$ & IV & $\begin{array}{l}\mathrm{B}^{*}(-)_{\mathrm{L}} \\
\mathrm{A}^{*}(-)_{\mathrm{BP}, \mathrm{ET}} \\
\mathrm{T}^{*}(-)_{\mathrm{M}}\end{array}$ \\
\hline Resveratrol & $\begin{array}{l}\text { Decrease LDL and increase HDL } \\
\text { Antioxidant } \\
\text { Anticancer }\end{array}$ & II & $\begin{array}{l}\mathrm{B}^{*}(-)_{\mathrm{S}} \\
\mathrm{A}^{*}(-)_{\mathrm{ET}} \\
\mathrm{T}^{*}(-)_{\mathrm{M}, \mathrm{G}}\end{array}$ \\
\hline EGCG & $\begin{array}{l}\text { Antioxidant, Antitumor } \\
\text { Anti-inflammatory }\end{array}$ & III & $\begin{array}{l}\mathrm{B}^{*}(-)_{\mathrm{L}, \mathrm{S}} \\
\mathrm{A}^{*}(-)_{\mathrm{TJ}, \mathrm{AT}, \mathrm{ET}} \\
\mathrm{T}^{*}(-)_{\mathrm{M}, \mathrm{C}}\end{array}$ \\
\hline
\end{tabular}

BCS - Biopharmaceutical Classification System; NuBACS - Nutraceutical Bioavailability Classification Scheme; LDL-low-density lipoprotein cholesterol; HDL high-density lipoprotein cholesterol; EGCG ( - )-epigallocatechin-3-gallate; $B *$ - bioaccessibility; $A *$ - absorption; $T^{*}$ - transformation; L-poor liberation from the food matrix; S- poor solubility in the gastrointestinal fluids; I- interactions that promote insolubility; TJ-poor transport through the mucus layer through the tight junctions, BP- poor transport through the bilayer membrane; AT-inhibition of active transporters, ET-presence of efflux transporters in the epithelium cells; C- changes in bioactivity due to chemical degradation; M-changes in bioactivity due to metabolism; + non-limiting factor; - limiting factor. the limited release from food matrix and the formation of insoluble complexes with other compounds in the GI tract (Arora \& Jaglan, 2016).

\subsection{Emerging strategies for improving nutraceuticals' bioavailability}

Some of the strategies that have emerged to overcome the limited bioavailability of most nutraceuticals are discussed below.

\subsubsection{Delivery systems' optimization}

The specific design of colloidal delivery systems is an efficient way to improve nutraceuticals' dispersibility, stability, food compatibility and bioavailability. In fact, bioavailability is known to depend on delivery systems' composition, size and interfacial properties, and so these properties can be optimized to develop effective delivery systems (McClements, 2015b). Different approaches could be used such as particle size reduction of the delivery system, incorporation of specific ingredients in the delivery systems' formulation known to increase the absorption of encapsulated nutraceuticals, or co-ingestion of nutraceuticals and excipient foods.

4.2.1.1. Nano-based formulations. An effective approach to improve the oral bioavailability of nutraceuticals is to use delivery systems at the nanoscale, due to the increase of the surface-to-volume ratio by reducing particle size into the nano-range (Faridi Esfanjani \& Jafari, 2016). The use of nano-delivery systems could facilitate the entry of the nutraceutical through biological barriers, while avoiding the metabolic modifications that lead to low absorption (Rein et al., 2013).

In recent years, different nano-delivery systems have been shown to boost bioavailability and efficacy of nutraceuticals. Parthasarathi et al. (2016) evaluated the effect of droplet size on oral vitamin E emulsions bioavailability and observed that nanoemulsions with a size of $277 \mathrm{~nm}$ showed a 3-fold increase in the AUC when compared to conventional emulsions (Parthasarathi et al., 2016). Evaluating the influence of particle size on $\beta$-carotene bioaccessibility, other authors have shown that $\beta$-carotene bioaccessibility increases with decreasing droplet diameter (small $>$ medium $>$ large) (Salvia-Trujillo, Qian, MartínBelloso, \& McClements, 2013). In another study, hydroxycitric acid (HCA) has been nanoencapsulated using SLNs. The authors observed that HCA-loaded nanoparticles exhibited a 2 -fold higher bioavailability than non-encapsulated HCA and a 1.3-fold higher bioavailability compared to microparticles due to smaller particle size, longer residence time and HCA controlled release (Ezhilarasi, Muthukumar, \& Anandharamakrishnan, 2016). In order to enhance curcumin absorption by oral administration, liposome-encapsulated curcumin has been developed (Takahashi, Uechi, Takara, Asikin, \& Wada, 2009). Results have shown a 5-fold increase in bioavailability of liposome-encapsulated curcumin compared to suspension (based on AUC). Other authors have showed that the bioavailability of coenzyme Q10 increased 5-fold after administration of the micellar NanoSolve formulation (Lipoid GmbH, Ludwigshafen, Germany), and the bioavailability of vitamin E was enhanced 10-fold, both compared to the pure substances (Wajda, Zirkel, \& Schaffer, 2007).

4.2.1.2. Mucus diffusion enhancers and absorption enhancers. The incorporation of specific compounds in the delivery systems formulation is another alternative to enhance the absorption of nutraceuticals. This can be achieved in two ways: by improving mucus diffusion of lipophilic compounds using mucolytic agents, or by improving their permeability using absorption enhancers (Gleeson et al., 2016).

Mucolytic agents or mucus diffusion enhancers have the ability to cleave mucoglycoprotein substructures responsible for the mucus layer viscoelastic and adhesive properties, facilitating the penetration of compounds to the underlying absorptive endothelial cells (Müller et al., 2012). Therefore, they hold the potential to increase intestinal 
absorption of nutraceuticals that are unable to penetrate the small intestinal mucus layer. N-acetylcysteine (NAC) (Yuan et al., 2015) and mucolytic enzymes such as bromelain and papain (Pereira de Sousa et al., 2015) are some examples of compounds that can be used as mucolytic agents.

Absorption enhancers, also termed permeation or bioavailability enhancers, are functional agents included in formulations to improve the absorption of a bioactive compound, usually by enhancing membrane permeation (Aungst, 2012). Compounds such as bile salts, surfactants, fatty acids, chelating agents, salicylates, polymers and herbal bioactives have shown to be effective in improving the intestinal absorption of different nutraceuticals (Kesarwani \& Gupta, 2013). They can act either paracellularly by the opening of $\mathrm{TJ}$ or, in most cases, transcellularly by increasing plasma membrane permeability, or a combination of both (Maher et al., 2016). For example, chitosan increases the absorption via the paracellular route by redistribution of the cytoskeletal F-actin, causing the opening of the TJ, whereas bile salts and fatty acids increase the absorption by improving hydrophobic bioactive compounds' solubility in the aqueous layer or by increasing the fluidity of apical and basolateral membranes (Kesarwani \& Gupta, 2013).

Although several absorption enhancers have been successful used by the pharmaceutical industry for development of formulations for drugs that have poor membrane permeation, most of them could cause membrane perturbation to varying extents. The majority of these damages are rapidly repairable; however, it is still unknown if the repeated dosing of some absorption enhancers for chronic therapy can cause irreversible epithelial damage (McCartney, Gleeson, \& Brayden, 2016). In order to reduce the risk of toxicity, permeation enhancers should reversibly disturb the intestinal barrier ideally without being absorbed, should be pharmacologically inert, have excipient or GRAS status, and have a history of safe use in humans (Gleeson et al., 2016). The use of bio-based permeation enhancers could be a safe and efficient alternative (Kesarwani \& Gupta, 2013). Table 4 shows examples of absorption enhancers that can be used in food formulations (GRAS status or of food origin) to promote nutraceuticals intestinal absorption.

\subsubsection{Food matrix design: excipient foods}

As an alternative to the incorporation of nutraceuticals in delivery systems, nutraceuticals may be delivered as part of food matrices, that can be divided into two major classes: functional foods and excipient foods (McClements, 2015a). Functional foods contain nutraceuticals trapped within the food matrix, whereas an excipient food can be defined as a component that may not exhibit bioactivity itself, but it may increase the efficacy of nutraceuticals that are co-ingested with it (McClements \& Xiao, 2014). In particular, excipient foods have gained much attention in the last years. In this case, nutraceuticals might be left in their natural source (e.g. fruit or vegetable) and their bioavailability can be boosted by consuming them with excipient foods, specifically formulated to control their release, solubilization, transport, metabolism and absorption within the GI tract (McClements, 2015a).

When designing an excipient food, different factors must be considered: i) the composition and structure of the food matrix should be specifically designed to allow an increase in the bioavailability of coingested nutraceuticals, ii) the excipient food should have good sensorial attributes, iii) a food matrix that can be consumed in a regular basis with foods containing nutraceuticals must be chosen, iv) the excipient food should have a sufficiently long shelf-life and be easy to storage (McClements \& Xiao, 2014). Salad dressings, sauces, yogurts, creams, ice-creams, butter and margarine are some examples of potential excipient foods. O/W emulsions are particularly suitable candidates for developing excipient foods due to the great flexibility in their design (e.g. ability to incorporate hydrophilic, amphiphilic and lipophilic ingredients, being their composition and structure easily altered), easy preparation, high physical stability, rapid GI digestibility and due to the fact that they are already used in food and beverage products (McClements et al., 2016).

The potential of emulsion-based excipient systems in enhancing the lipophilic nutraceuticals' bioavailability in fruits and vegetables has been highlighted in several works. However, it has been also showed that excipient emulsions' efficacy to increase nutraceuticals bioavailability depends on different factors such as nutraceutical itself, lipid phase, emulsifier type, emulsions' size or thermal treatment. The influence of the initial droplet size on the ability of excipient emulsions to increase carotenoid bioaccessibility from carrots has been investigated (Zhang, Zhang, Zou et al., 2016). Authors shown that carotenoid bioaccessibility significantly increased with the decrease of lipid droplet size in the excipient emulsions, being this result attributed to the rapid formation of mixed micelles that solubilize the carotenoids in the intestinal fluids.

In another work, the use of excipient emulsions with different droplet sizes to increase the lycopene bioaccessibility in tomato juice has been evaluated (Salvia-Trujillo \& McClements, 2016). Lycopene bioaccessibility increased from $7.5 \%$ (emulsion-free samples) to $12.5 \%$ and $10 \%$ for emulsions containing small and large droplets, respectively. The authors attributed this relatively modest increase in bioaccessibility to the high level of lycopene entrapment within the chromoplasts, which remained intact during in vitro digestion, thus preventing the transfer of lycopene into the mixed micelles.

Other authors developed zein nanoparticles encapsulating curcumin to obtain a high loading capacity and good chemical stability. They mixed these nanoparticles with digestible lipid nanoparticles developed to increase curcumin bioaccessibility. It has been concluded that delivery systems containing mixed colloidal particles (i.e. protein and lipid nanoparticles) are able to increase the lipophilic bioactive compounds' bioaccessibility (Zou et al., 2016).

These examples show that nutraceuticals' bioavailability can be increased by the addition of digestible lipids that form mixed micelles after digestion, and solubilize nutraceuticals within intestinal fluids. This strategy can be applied to nutraceuticals whose bioavailability is low due to their limited release from the food matrix and their limited solubility in intestinal fluids (i.e. $B^{*}(-)_{\mathrm{L}, \mathrm{S}}$ compounds). In the case of nutraceuticals whose bioavailability is low due to other factors, such as GI transformation or poor absorption (i.e. $A^{*}(-)$ compounds), one of the strategies that could be adopted is the design of excipient foods formulated with food grade components that increase cell permeability or reduce efflux mechanisms, such as piperine (Kesarwani \& Gupta, 2013) or sucrose esters (Szúts \& Szabó-Révész, 2012).

Although excipient food technology could be used to create new food products specifically developed to enhance nutraceuticals' bioavailability in natural products, it is also important to evaluate the safety of these products before their widespread application. In fact there are some concerns that must be addressed: i) besides increasing the nutraceutical bioavailability, the excipient food may also enhance the bioavailability of any toxic agents present in a food product; ii) by increasing the nutraceutical bioavailability, the excipient food may change its toxicity profile; iii) the alteration of the barrier properties of cell membranes may promote dysfunction of the intestinal barrier and iv) the nature of the gut microbiota could also be altered by changing the nutraceuticals' type and concentration reaching the colon (McClements et al., 2016).

\section{Evaluation of bioaccessibility and bioavailability of nutraceuticals within nano-delivery systems}

Delivery systems for nutraceuticals, such as nano-delivery systems, are promising structures to overcome the limitations of nutraceuticals after oral intake (e.g. poor aqueous solubility and poor oral bioavailability) (Acosta, 2009). Consequently, new nano-based approaches emerged and demonstrated their potential to enhance nutraceuticals' bioavailability. Several in vitro and in vivo studies have been conducted to assess the bioaccessibility and bioavailability of nutraceuticals within 


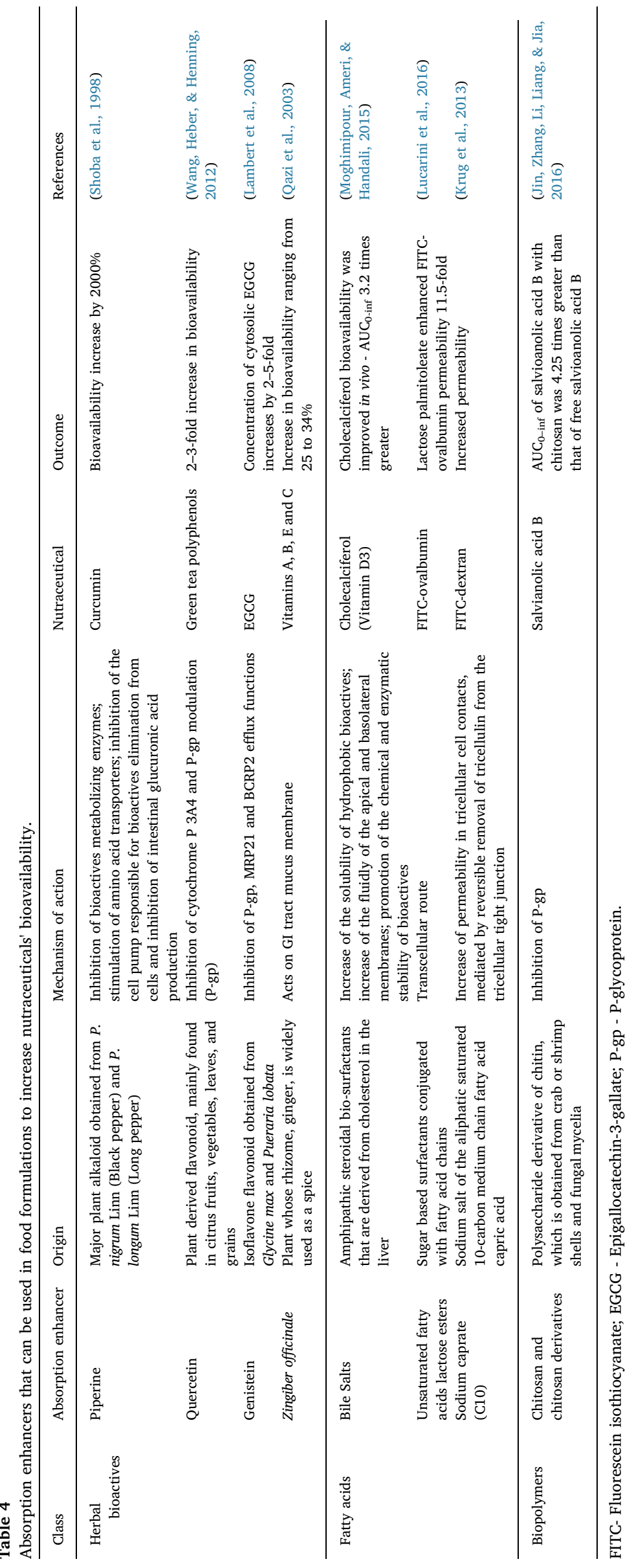


nano-delivery systems. Therefore, these topics related with nano-delivery systems will be described and discussed below.

\subsection{In vitro}

In the light of major concerns of nutraceutical nano-based formulations related to their bioavailability and stability during the digestive process, several in vitro studies, ranging from measurements of bioaccessibility to the studies of uptake in cell culture models, have been proposed. Distinct in vitro model systems are described below.

\subsubsection{Artificial in vitro gastrointestinal systems}

The bioaccessibility of a nutraceutical is determined by measuring the quantity of ingested dose (with intact structure) reaching the absorption site. To verify the bioaccessibility of a nutraceutical, numerous in vitro models can be used to simulate GI tract environment (Ting, Zhao, Xia, \& Huang, 2015). In fact, in vitro models generally differ in the inclusion of different stages of digestion (i.e. mouth, gastric, small intestinal and large intestinal); digestion times; concentrations of electrolytes; bile acids' $\mathrm{pH}$; digestive enzymes involved and peristalsis motion. In vitro models can be static (i.e. with prefixed concentrations and volumes of digested materials, enzymes, salts, etc.) or dynamic (i.e. mimic the continuous changes of the physicochemical conditions) (Kostewicz et al., 2014). The pH-stat model is an example of a static model which simulates the digestion environment in the small intestine. This model is an especially appropriate apparatus to assess the change in bioaccessibility of hydrophobic compounds (Kostewicz et al., 2014). For digestion of nano-delivery systems, a multiple-step technique is normally used to model the physicochemical transformations which these systems undergo as they pass from an organ to the other (e.g. stomach to intestine) in the GI tract (Alger, Momcilovic, Carlander, \& Duncan, 2014). For instance, a digestion model involving the in vitro simulation of stomach, duodenum, jejunum and ileum has been used to evaluate the digestion of curcumin nanoemulsions (Pinheiro et al., 2013) and $\beta$-lactoglobulin nanohydrogels containing riboflavin (Madalena et al., 2016). However, static models cannot reproduce the in vivo physical conditions including the GI tract peristalsis motion, integration of all of the influential physiological factors, transitional change in the physiological environment, and elimination from the absorption site. Dynamic models simulating the GI digestion include more complex and precise models for nutraceuticals' bioaccessibility estimation, which is the case of the dynamic TNO gastro-Intestinal Model (TIM-1) (Sjögren et al., 2014). This continuous in vitro model offers control of physiological parameters that simulates relevant aspects of digestion (e.g. peristalsis motion, gastric and intestinal residence time). Also, bioaccessible fraction can be sampled continuously from jejunum and ileum segments at programmed times. As a result, intestinal bioaccessibility of compounds in different types of formulations can be investigated (Ting et al., 2015). Bourbon et al. (2016) evaluated the digestibility of chitosan-coated lactoferrin-glycomacropeptide (Lf-GMP) nanohydrogels using a dynamic in vitro system. These authors observed that chitosan improved the stability of Lf and GMP (i.e. these proteins were hydrolyzed at a slower rate) and maintained their structure intact for longer period of time (Bourbon et al., 2016).

\subsubsection{Intestinal epithelial monolayer assays (cellular uptake)}

To further investigate the transport of nano-delivery systems through the natural barriers of the GI surface, a variety of in vitro approaches are available (Shahbazi \& Santos, 2013). In vitro cell lines (monocultures), such as Caco-2 and HT-29 have been frequently used (Gamboa \& Leong, 2013) due to its similar morphology (i.e. organized brush borders, functional $\mathrm{TJ}$ between adjacent cells, enzymes, nuclear receptors and transporters expressed in membrane) with intestinal human epithelial cells, known as enterocytes (Song et al., 2013). Caco-2 (human colon carcinoma) cell line is grown normally as a monolayer on polycarbonate membrane in transwell chambers. In this system, the apical and basolateral culture fluids are separated allowing the transport of molecules from one culture fluid to the other (Liu et al., 2016). This model is useful to quantify compound absorption across the monolayer, determine apparent permeability coefficient $\left(P_{a p p}\right)$ and analyze uptake mechanisms (e.g. absorption or diffusion). When using in vitro cell lines that simulate intestinal epithelium, motorization of transepithelial electrical resistance (TEER) and fluorescent marker transport (e.g. fluorescein) during transport assay are common methods to assure the integrity of the monolayers (Lorkowski, 2012). Recently, Caco-2 cell line was used to explore transport mechanisms of two different structures: polymeric PLGA nanoparticles (He et al., 2013) and curcumin loaded-folic acid/soy protein nanoparticles (Teng, Luo, Wang, Zhang, \& Wang, 2013). In another study, (-)-epigallocatechin3-gallate (EGCG) in ovoalbumin-dextran conjugate nanoparticles showed higher $P_{a p p}$ on Caco-2 monolayers compared to free EGCG in solution (Li \& Gu, 2014). As for HT29 cells (being the clone HT29-H of particular interest), this specific cell line has the ability of producing mucus (Schimpel et al., 2014), a variable that is mostly neglected in other in vitro based-cell models. Mucus is crucial to properly study transport mechanisms, mainly because its presence can hamper the diffusion of nano-delivery systems before they can reach intestinal cells (Guri et al., 2013; Plapied et al., 2011). Also, it is known that specialized M-cells are involved in nano-delivery systems' transport; in order to mimic this feature, human Raji B lymphocyte cells are being cocultured with other cell lines (e.g. Caco-2 cells), once these cells exhibit $\mathrm{B}$ cell markers that induced the reconstruction of M cells (des Rieux et al., 2007).

It is important to highlight that the successful prediction of nutraceuticals and nano-delivery systems absorption highly depend on the ability of in vitro models in simulating the characteristics of the in vivo intestinal epithelium. Thus, in order to overcome the individual limitations of each cell type, and to accurately predict the transport mechanism of nano-delivery systems, in vitro models integrating different cell types (co-cultures) are being used (Antunes, Andrade, Araújo, Ferreira, \& Sarmento, 2013; Schimpel et al., 2014). Examples are the co-culture of Caco-2 and HT29-MTX cells to assess the transport route of curcumin-solid lipid nanoparticles (Guri et al., 2013), and a triple coculture Caco-2/HT29/Raji B cells with the aim of developing a more accurate in vitro model of the intestinal epithelial layer (Lopes et al., 2016). An overview of the in vitro cellular uptake studies is given in Table 5.

\subsection{In vivo}

In vitro models or a combination of models to predict the efficacy of nano-delivery systems are valuable tools to study the factors that impact nutraceuticals bioaccessibility and bioavailability. Still, the complex simulation of pre-absorption (e.g. TIM-1 system) and absorption (e.g. Caco-2 and HT29-MTX co-culture) using in vitro models cannot entirely reproduce and predict the oral bioavailability due to lack of biological factors, such as systemic metabolism and volume of body distribution (Gamboa \& Leong, 2013).

Many research studies implemented in vivo models to investigate the beneficial effects of nutraceuticals or pharmacokinetics of nano-delivery systems. In vivo pharmacokinetic studies, are considered the best procedure to predict human oral bioavailability although they are timeconsuming, costly and there are often ethical constrains involved. In vivo animal models (e.g. mouse, rat, sheep and pig) present all the physiological and physiochemical events during the absorption, distribution, metabolism and elimination (ADME) of ingested nano-delivery systems and nutraceuticals. Some in vivo studies investigated body distribution of nano-delivery systems or nutraceuticals following oral delivery using diverse detection techniques. Determination of nutraceuticals and their metabolites in the animal blood, tissues, urine or the enzymes levels may provide data of its bioavailability (Gamboa \& Leong, 2013). The use of radio and stable isotopic techniques for some 


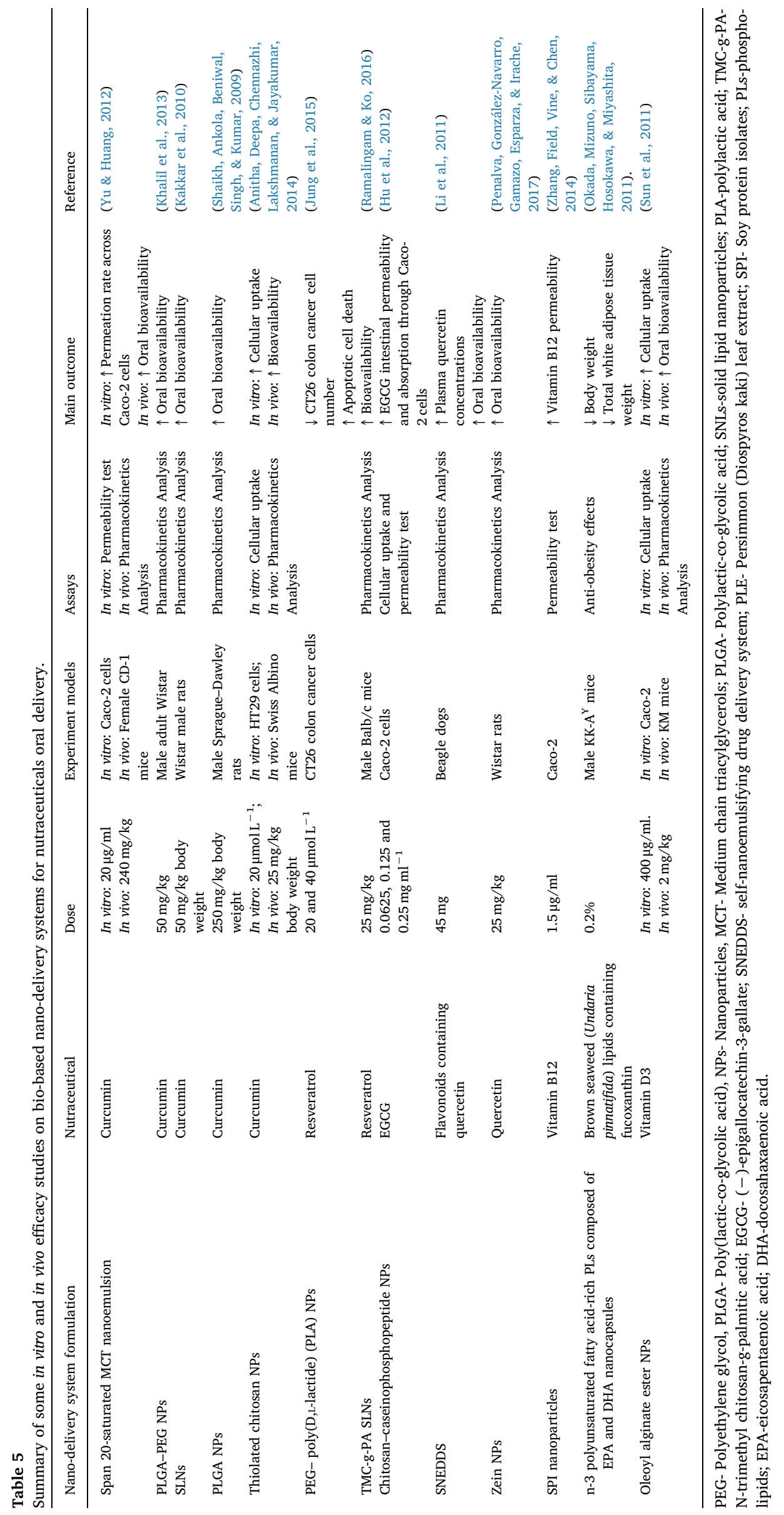




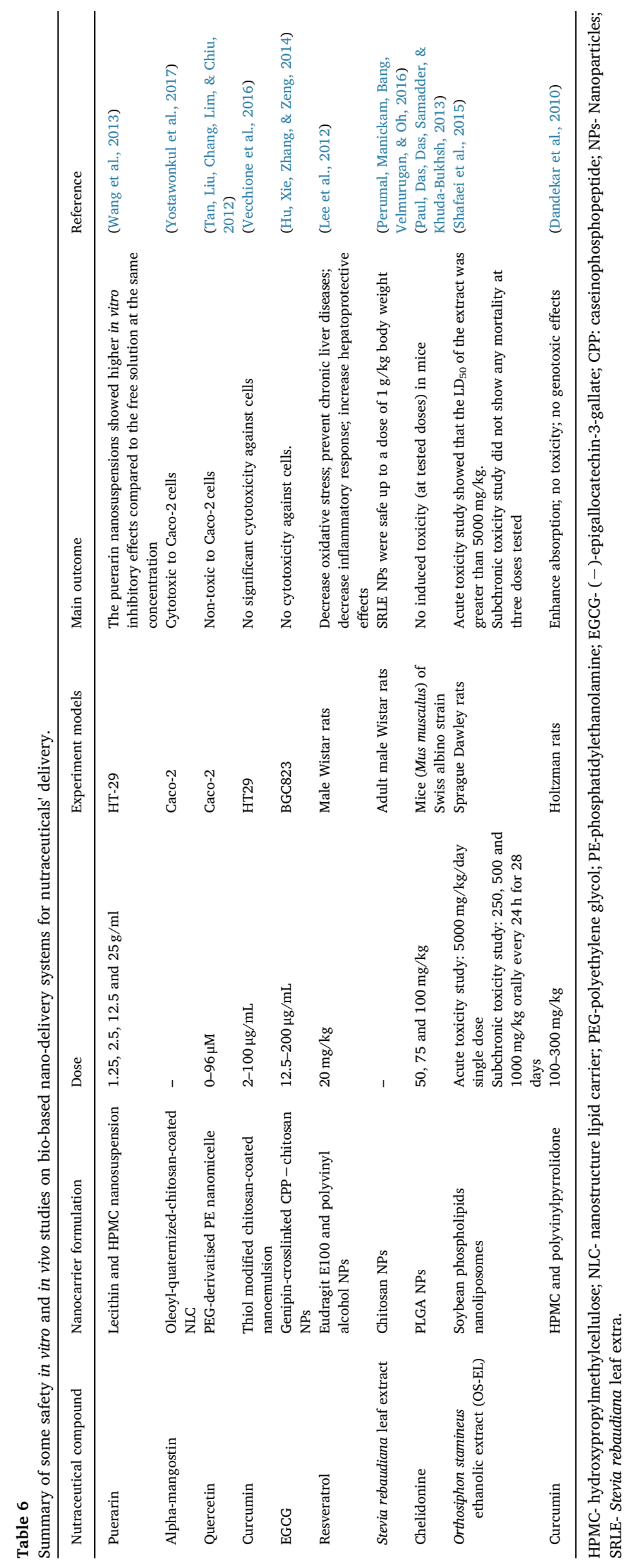


nutraceuticals or nano-delivery systems allows the examination of its route, tissue distribution and bioavailability. Sonaje et al. (2012) studied the biodistribution of isotope-labelled insulin loaded chitosan/poly $(\gamma$-glutamic acid) nanoparticles following the oral administration using microscopic and computed-tomographic approaches. The in vivo results indicated that insulin could be absorbed into the systemic circulation, being most of chitosan retained in the microvilli scaffolds (Sonaje et al., 2012).

In general, nano-delivery systems are retained in the GI tract with some distribution reported in the liver, bladder, kidney and lymph nodes. ADME of orally administered nanomaterials is influenced by their characteristics such as shape, size, hydrophobility, surface charge, and functionalized groups (Griffin et al., 2016). Selected in vivo studies are reported in Table 5. However, in vivo pharmacokinetic data published are rarely comparable since animal models, dose, formulation, and delivery system are selected without harmony.

\subsection{In situ and ex vivo}

In situ and ex vivo models are also widely used to replicate the in vivo situation, once they allow a more accurate prediction of compounds' absorption comparing to in vitro models.

In situ experiments include intestinal loop, intestinal perfusion and intestinal vascular cannulation models. They typically refer to experiments on whole animals, providing intact intestinal mucosa, complete blood supply and nerve domination, together with expression of enzymes and transporters (Liu et al., 2016). However, sophisticated surgical procedures and instrumentation are required. In these methods, the compounds' absorption can be directly measured by the disappearance of the absorbed compound from the gut or indirectly measured by the rate of compounds' appearance in plasma, excretion in urine, or by the speed of onset or degree of pharmacologic action (Luo et al., 2013). For example, in situ intestinal perfusion method has been used to study the potential enhancement of curcumin's intestinal absorption and oral bioavailability via taurocholic acid-modified NLCs. It has been demonstrated that the addition of taurocholic acid to the NLCs boosted curcumin absorption. In fact, depending on the degree of modification, curcumin taurocholic acid-modified NLCs displayed about 5-15-fold higher area under the curve in rats after oral administration compared to unmodified curcumin NLCs (Tian et al., 2017).

Ussing chamber and everted intestinal sac methods are the most common examples of ex vivo studies. They are advantageous methods because the cell-cell contact remain intact, all cell types remain present, being enzyme systems, co-factors and transporters present in their physiological context (Esther, Inge, \& Geny, 2007). However, they present some drawbacks such as interruption of the normal blood flow and lack of a nervous system, which sometimes lead to an inappropriate estimation of oral absorption degree (Luo et al., 2013). Despite that, ex vivo methods are simple and widely used in the design and testing of encapsulated nutraceuticals. For example, nanoparticles based on quaternary ammonium-chitosan conjugate have been tested as vehicles for antioxidants contained in red grape seeds extracts, being the Ussing chamber the method applied to study the nanoparticles' penetration across intestinal barrier. The nanoparticles have been found to migrate from the donor to the acceptor compartment and penetrate across excised rat intestine from mucosal to serosal side in an integral state, showing great potential as profitable vehicles for oral administration of antioxidants extracts (Fabiano et al., 2016).

\section{Safety evaluation of nano-delivery systems}

The great potential of nano-based formulations for food applications introduced serious concerns about their toxicity. This concern is mainly based on their increased surface-to-volume ratio compared to their micro-sized counterparts. Potential in vitro and in vivo effects of nanobased formulations cannot be anticipated from a previous knowledge of bulk analogs. So, it is essential to consider relevant parameters to study toxicity (Lai, 2012). As a starting point to toxicity assessment, it is fundamental to characterize the nanostructure itself. For example, determine its materials, structure, size, shape, surface area, charge, solubility, hydrophobicity, stability and aggregation state (Podila \& Brown, 2013). There are numerous studies of toxicity measurement in various cellular and animal models using different techniques. A selection of in vitro and in vivo toxicity evaluation using nano-delivery systems as carriers of nutraceuticals is summarized in Table 6.

Toxicity tests are mainly focused on in vitro toxicity analysis, which includes cellular uptake and localization studies (using microscopy methods), cellular viability assays (cellular proliferation, necrosis and apoptosis), and mechanistic assays measuring DNA damage and oxidative stress (Arora, Rajwade, \& Paknikar, 2012). These assays are generally used once they are easy, efficient and low cost. The limitations of these assays are the fact of the results can vary depending on the type of assay and nano-based formulations used (e.g. dose concentration), and consequently could not predict in vivo toxicity (Arora et al., 2012). In vivo toxicity assays are normally conducted in rat and mouse models. These models offer an opportunity to study nanomaterial acute and chronic dose-response, biodistribution, retention, excretion, and other effects. The most used animal toxicity measurements comprise tissue morphology, changes in cell population, blood serum chemistry, weight change, and longevity. Nevertheless, it is fundamental to consider other variables such as dose concentration, and targeted tissues or organs. Even though rat and mouse models could be used to analyze a complete organism, some caution have to be considered when extrapolating toxicity results for human consumption. In addition, biodistribution quantification frequently needs fluorescent or radiologic labels, which may alter the clearance route. Currently, there are no standardized protocols available for particular nanomaterials, which led to numerous studies with different preparation methods, materials, cell lines, doses, etc. making difficult to compare results.

Many of the recently developed nano-delivery systems can increase the transport of poorly hydrophilic compounds across the GI barrier by enhancing paracellular transport through $\mathrm{TJ}$ opening. Certain nanodelivery systems' components such as surfactants, polymers, chelating agents, may increase the gap dimensions and consequently permeability (Maher et al., 2016). The use of selected enhancers that alter barrier integrity or permeability could induce toxicity, even though many compounds could be considered GRAS, due to the continuous perturbation of the intestinal epithelia through mild detergent-based surfactant effects (Chassaing et al., 2015). Also, selected enhancers could stimulate permeability to inflammatory molecules (such as allergens, toxins and bacteria) which damage colonic epithelium and consequently, may lead to intestinal inflammation diseases such as inflammatory bowel disease (Maher et al., 2016). Therefore, the absorption enhancer action should be reversible (i.e. damage quickly repaired) and their action should be rapid, coinciding with the presence of the nutraceutical at the absorption site.

Many components in nano-delivery systems, such as antibody fragments, peptides, proteins, can act as antigens, resulting in increased immunotoxicity. Additionally, consumers could take high amounts of nano-delivery systems containing surfactants that may cause adverse effects in the body (Renukuntla et al., 2013). Clinical trials could be used to assess the short-term nano-delivery systems' toxicity, as well as long-term toxicity because chronic exposure and accumulation could be a serious problem (Elsaesser \& Howard, 2012). Biodegradable nanodelivery systems can be degraded or digested in GI tract. Nutraceuticals encapsulated within those nano-delivery systems could be also absorbed; however, their characteristics, structure and pharmacokinetic may be changed. If the objective is to decrease toxicity and adverse effects of nano-delivery systems, target ligants could be used in their surface to targeted specific cells (Ciappellano, Tedesco, Venturini, \& Benetti, 2016). If the nutraceutical is incorporated into a food product that is consumed in a regular basis and in large quantities (for example, 
beverages), this is very important. Presently, there is a lack of guidelines and standardized safety tests variables established to determine toxicity and adverse effects of nano-delivery systems (GarduñoBalderas, Urrutia-Ortega, Medina-Reyes, \& Chirino, 2015). Being so, as previously mentioned, their safety concerns should be explored and food safety studies should be conducted about ADME and toxicological characteristics.

\section{Future trends and conclusions}

The incorporation of nutraceuticals into food products provides a simple way of developing a variety of functional foods that could help fulfilling some of the consumers' health and wellness needs. However, the nutraceuticals' effectiveness depends on preserving their bioavailability. Delivery systems can be specifically designed to modulate bioaccessibility, absorption, or transformation profile of nutraceuticals in the GI tract, boosting their bioavailability and consequently, their health benefits. Progress is being made by transferring the knowledge from pharmaceutical applications, including the use of nano-delivery systems, absorption enhancers or excipient foods that have been shown to improve solubility, stability or permeability of nutraceuticals. However, there are still some challenges that must be overcome, that include: i) the exclusive use of food-grade ingredients, ii) development of delivery systems stable in food matrices along processing and shelflife, iii) difficulty to control the release of nutraceuticals, iv) optimization of delivery systems formulations in order to increase the permeability through the intestinal walls, v) potential toxicity of absorption enhancers due to perturbation of the intestinal epithelia even for agents with GRAS status, and vi) in the case of nano-delivery systems, the risk assessment of their use of in food. This latter issue is particularly relevant, once, although the absorption of intact food grade delivery systems into the systemic circulation is unlikely due to changes after undergone gastric and intestinal digestion, reliable data regarding the risks of nano-delivery systems cannot be found in the literature and also, from the existent studies, it is not possible to predict the consequences of their long-term intake. Therefore, in order to evaluate their safety, more realistic in vitro digestive models need to be designed to undoubtedly understand the fate of micro and nano-delivery systems/nutraceuticals in the GI tract; more advanced analytical methods to accurately detect and quantify nano-delivery systems must be developed; and a clear legislation and guidelines concerning their impact on human health and on environment must be assured. Also, in order to develop delivery systems with increased functionality, more efforts should be made towards understanding the interactions between the delivery systems and the food matrix. Future studies should focus on improving the physicochemical characteristics of the delivery systems, by the careful selection of the delivery systems' formulation on a caseby-case basis.

The development of delivery systems that afford nutraceuticals' greatest functionality and safety may create opportunities to apply enabling technologies such as nanotechnology more broadly in the food industry.

\section{Acknowledgements}

Ana C. Pinheiro and Joana T. Martins acknowledge the Foundation for Science and Technology (FCT) for their fellowships (SFRH/BPD/ 101181/2014 and SFRH/BPD/89992/2012). This work was supported by Portuguese Foundation for Science and Technology (FCT) under the scope of the Project PTDC/AGR-TEC/5215/2014, of the strategic funding of UID/BIO/04469/2013 unit and COMPETE 2020 (POCI-010145-FEDER-006684) and BioTecNorte operation (NORTE-01-0145FEDER-000004) funded by the European Regional Development Fund under the scope of Norte2020 - Programa Operacional Regional do Norte.

\section{References}

Abaee, A., Mohammadian, M., \& Jafari, S. M. (2017). Whey and soy protein-based hydrogels and nano-hydrogels as bioactive delivery systems. Trends in Food Science \& Technology, 70, 69-81.

Acevedo-Fani, A., Soliva-Fortuny, R., \& Martín-Belloso, O. (2018). Photo-protection and controlled release of folic acid using edible alginate/chitosan nanolaminates. Journal of Food Engineering, 229, 72-82.

Acosta, E. (2009). Bioavailability of nanoparticles in nutrient and nutraceutical delivery. Current Opinion in Colloid \& Interface Science, 14, 3-15.

Aditya, N. P., Espinosa, Y. G., \& Norton, I. T. (2017). Encapsulation systems for the delivery of hydrophilic nutraceuticals: Food application. Biotechnology Advances, 35, 450-457.

van Aken, G. A. (2010). Relating food emulsion structure and composition to the way it is processed in the gastrointestinal tract and physiological Responses: What are the opportunities? Food Biophysics, 5, 258-283.

van Aken, G. A., Bomhof, E., Zoet, F. D., Verbeek, M., \& Oosterveld, A. (2011). Differences in in vitro gastric behaviour between homogenized milk and emulsions stabilised by Tween 80, whey protein, or whey protein and caseinate. Food Hydrocolloids, 25, $781-788$.

Alexander, M., Acero Lopez, A., Fang, Y., \& Corredig, M. (2012). Incorporation of phytosterols in soy phospholipids nanoliposomes: Encapsulation efficiency and stability. LWT - Food Science and Technology, 47, 427-436.

Alger, H., Momcilovic, D., Carlander, D., \& Duncan, T. V. (2014). Methods to evaluate uptake of engineered nanomaterials by the alimentary tract. Comprehensive Reviews in Food Science and Food Safety, 13, 705-729.

Amidon, G. L., Lennernäs, H., Shah, V. P., \& Crison, J. R. (1995). A theoretical basis for a biopharmaceutic drug Classification: The correlation of in vitro drug product dissolution and in vivo bioavailability. Pharmaceutical Research, 12, 413-420.

Anitha, A., Deepa, N., Chennazhi, K. P., Lakshmanan, V.-K., \& Jayakumar, R. (2014). Combinatorial anticancer effects of curcumin and 5-fluorouracil loaded thiolated chitosan nanoparticles towards colon cancer treatment. Biochimica et Biophysica Acta (BBA) - General Subjects, 1840, 2730-2743.

Antunes, F., Andrade, F., Araújo, F., Ferreira, D., \& Sarmento, B. (2013). Establishment of a triple co-culture in vitro cell models to study intestinal absorption of peptide drugs. European Journal of Pharmaceutics and Biopharmaceutics, 83, 427-435.

Arora, D., \& Jaglan, S. (2016). Nanocarriers based delivery of nutraceuticals for cancer prevention and treatment: A review of recent research developments. Trends in Food Science \& Technology, 54, 114-126.

Arora, S., Rajwade, J. M., \& Paknikar, K. M. (2012). Nanotoxicology and in vitro studies: The need of the hour. Toxicology and Applied Pharmacology, 258, 151-165.

Ashraf, O., Nasr, M., Nebsen, M., Said, A. M. A., \& Sammour, O. (2018). In vitro stabilization and in vivo improvement of ocular pharmacokinetics of the multi-therapeutic agent baicalin: Delineating the most suitable vesicular systems. International Journal of Pharmaceutics, 539(1-2), 83-94.

Augustin, M. A., \& Hemar, Y. (2009). Nano- and micro-structured assemblies for encapsulation of food ingredients. Chemical Society Reviews, 38, 902-912.

Augustin, M. A., \& Sanguansri, L. (2008). Encapsulation of bioactives. In J. M. Aguilera, \& P. J. Lillford (Eds.). Food materials science: Principles and Practice (pp. 577-601). New York, NY: Springer New York.

Augustin, M. A., \& Sanguansri, L. (2012). Challenges in developing delivery systems for food additives, nutraceuticals and dietary supplements.

Aungst, B. J. (2012). Absorption Enhancers: Applications and advances. The AAPS Journal, 14, 10-18.

Babazadeh, A., Ghanbarzadeh, B., \& Hamishehkar, H. (2016). Novel nanostructured lipid carriers as a promising food grade delivery system for rutin. Journal of Functional Foods, 26, 167-175.

Bai, L., \& McClements, D. J. (2016). formation and stabilization of nanoemulsions using biosurfactants: Rhamnolipids. Journal of Colloid and Interface Science, 479, 71-79.

Basit, A. W. (2005). Advances in colonic drug delivery. Drugs, 65, 1991-2007.

Bawa, P., Pillay, V., Choonara, Y. E., \& Toit, L. C. d (2009). Stimuli-responsive polymers and their applications in drug delivery. Biomedical Materials, 4, 022001.

Berens, A. R., \& Hopfenberg, H. B. (1978). Diffusion and relaxation in glassy polymer powders. 2. Separation of diffusion and relaxation parameters. Polymer, 19, 489-496.

Bischoff, S. C., Barbara, G., Buurman, W., Ockhuizen, T., Schulzke, J.-D., Serino, M., et al. (2014). Intestinal permeability - a new target for disease prevention and therapy. BMC Gastroenterology, 14

Boegh, M., \& Nielsen, H. M. (2015). Mucus as a barrier to drug delivery - understanding and mimicking the barrier properties. Basic and Clinical Pharmacology and Toxicology, $116,179-186$.

Bohn, T., McDougall, G. J., Alegría, A., Alminger, M., Arrigoni, E., Aura, A.-M., et al. (2015). Mind the gap-deficits in our knowledge of aspects impacting the bioavailability of phytochemicals and their metabolites-a position paper focusing on carotenoids and polyphenols. Molecular Nutrition \& Food Research, 59, 1307-1323.

Bourbon, A. I., Cerqueira, M. A., \& Vicente, A. A. (2016). Encapsulation and controlled release of bioactive compounds in lactoferrin-glycomacropeptide nanohydrogels: Curcumin and caffeine as model compounds. Journal of Food Engineering, 180, $110-119$.

Chassaing, B., Koren, O., Goodrich, J., Poole, A., Srinivasan, S., Ley, R. E., et al. (2015). Dietary emulsifiers impact the mouse gut microbiota promoting colitis and metabolic syndrome. Nature, 519, 92-96.

Chatzidaki, M. D., Mitsou, E., Yaghmur, A., Xenakis, A., \& Papadimitriou, V. (2015). Formulation and characterization of food-grade microemulsions as carriers of natural phenolic antioxidants. Colloids and Surfaces A: Physicochemical and Engineering Aspects, 483, 130-136. 
Chen, M.-C., Mi, F.-L., Liao, Z.-X., Hsiao, C.-W., Sonaje, K., Chung, M.-F., et al. (2013). Recent advances in chitosan-based nanoparticles for oral delivery of macromolecules. Advanced Drug Delivery Reviews, 65, 865-879.

Chung, C., Sher, A., Rousset, P., Decker, E. A., \& McClements, D. J. (2017). Formulation of food emulsions using natural emulsifiers: Utilization of quillaja saponin and soy lecithin to fabricate liquid coffee whiteners. Journal of Food Engineering, 209, 1-11.

Ciappellano, S. G., Tedesco, E., Venturini, M., \& Benetti, F. (2016). In vitro toxicity assessment of oral nanocarriers. Advanced Drug Delivery Reviews, 106, 381-401.

Crank, J. (1975). The mathematics of diffusion (2 ed.). Oxford: Clarendon Press.

Dandekar, P. P., Jain, R., Patil, S., Dhumal, R., Tiwari, D., Sharma, S., et al. (2010). Curcumin-loaded hydrogel Nanoparticles: Application in anti-malarial therapy and toxicological evaluation. Journal of Pharmaceutical Sciences, 99, 4992-5010.

Daugherty, A. L., \& Mrsny, R. J. (1999). Transcellular uptake mechanisms of the intestinal epithelial barrier Part one. Pharmaceutical Science \& Technology Today, 4, 144-151.

Davitt, C. J. H., \& Lavelle, E. C. (2015). Delivery strategies to enhance oral vaccination against enteric infections. Advanced Drug Delivery Reviews, 91, 52-69.

Dima, Ş., Dima, C., \& Iordăchescu, G. (2015). Encapsulation of functional lipophilic food and drug biocomponents. Food Engineering Reviews, 7, 417-438.

Elsaesser, A., \& Howard, C. V. (2012). Toxicology of nanoparticles. Advanced Drug Delivery Reviews, 64, 129-137.

Esther, G. v. d. K., Inge, A. M. d. G., \& Geny, M. M. G. (2007). Vitro methods to study intestinal drug metabolism. Current Drug Metabolism, 8, 658-675.

Etcheverry, P., Grusak, M. A., \& Fleige, L. E. (2012). Application of in vitro bioaccessibility and bioavailability methods for calcium, carotenoids, folate, iron, magnesium, polyphenols, zinc and vitamins B6, B12, D, and E. Frontiers in Physiology, 3.

Ezhilarasi, P. N., Muthukumar, S. P., \& Anandharamakrishnan, C. (2016). Solid lipid nanoparticle enhances bioavailability of hydroxycitric acid compared to a microparticle delivery system. RSC Advances, 6, 53784-53793.

Fabiano, A., Mattii, L., Braca, A., Felice, F., Di Stefano, R., \& Zambito, Y. (2016). Nanoparticles based on quaternary ammonium-chitosan conjugate: A vehicle for oral administration of antioxidants contained in red grapes. Journal of Drug Delivery Science and Technology, 32, 291-297.

Fang, Z., \& Bhandari, B. (2010). Encapsulation of polyphenols - a review. Trends in Food Science \& Technology, 21, 510-523.

Faridi Esfanjani, A., Assadpour, E., \& Jafari, S. M. (2018). Improving the bioavailability of phenolic compounds by loading them within lipid-based nanocarriers. Trends in Food Science \& Technology, 76, 56-66.

Faridi Esfanjani, A., \& Jafari, S. M. (2016). Biopolymer nano-particles and natural nanocarriers for nano-encapsulation of phenolic compounds. Colloids and Surfaces B: Biointerfaces, 146, 532-543.

Fathi, M., Mozafari, M. R., \& Mohebbi, M. (2012). Nanoencapsulation of food ingredients using lipid based delivery systems. Trends in Food Science \& Technology, 23, 13-27.

Fuciños, C., Fuciños, P., Míguez, M., Katime, I., Pastrana, L. M., \& Rúa, M. L. (2014). Temperature- and $\mathrm{pH}$-sensitive nanohydrogels of poly( $\mathrm{N}$-isopropylacrylamide) for food packaging Applications: Modelling the swelling-collapse behaviour. PLoS One, 9, e87190.

Fundueanu, G., Constantin, M., Ascenzi, P., \& Simionescu, B. (2010). An intelligent multicompartmental system based on thermo-sensitive starch microspheres for temperature-controlled release of drugs. Biomedical Microdevices, 12, 693-704.

Gamboa, J. M., \& Leong, K. W. (2013). In vitro and in vivo models for the study of oral delivery of nanoparticles. Advanced Drug Delivery Reviews, 65, 800-810.

Garduño-Balderas, L. G., Urrutia-Ortega, I. M., Medina-Reyes, E. I., \& Chirino, Y. I. (2015). Difficulties in establishing regulations for engineered nanomaterials and considerations for policy makers: Avoiding an unbalance between benefits and risks. Journal of Applied Toxicology, 35, 1073-1085.

Gleeson, J. P., Ryan, S. M., \& Brayden, D. J. (2016). Oral delivery strategies for nutraceuticals: Delivery vehicles and absorption enhancers. Trends in Food Science \& Technology, 53, 90-101.

Griffin, B. T., Guo, J., Presas, E., Donovan, M. D., Alonso, M. J., \& O'Driscoll, C. M. (2016). Pharmacokinetic, pharmacodynamic and biodistribution following oral administration of nanocarriers containing peptide and protein drugs. Advanced Drug Delivery Reviews, 106, 367-380.

Guri, A., Gülseren, I., \& Corredig, M. (2013). Utilization of solid lipid nanoparticles for enhanced delivery of curcumin in cocultures of HT29-MTX and Caco-2 cells. Food \& Function, 4, 1410

Ha, T. V., Kim, S., Choi, Y., Kwak, H. S., Lee, S. J., Wen, J., et al. (2015). Antioxidant activity and bioaccessibility of size-different nanoemulsions for lycopene-enriched tomato extract. Food Chemistry, 178, 115-121.

Hariharan, S., Bhardwaj, V., Bala, I., Sitterberg, J., Bakowsky, U., \& Ravi Kumar, M. N. V. (2006). Design of estradiol loaded PLGA nanoparticulate formulations: A potential oral delivery system for Hormone therapy. Pharmaceutical Research, 23, 184-195.

He, B., Lin, P., Jia, Z., Du, W., Qu, W., Yuan, L., et al. (2013). The transport mechanisms of polymer nanoparticles in Caco-2 epithelial cells. Biomaterials, 34, 6082-6098.

Hu, B., Ting, Y., Yang, X., Tang, W., Zeng, X., \& Huang, Q. (2012). Nanochemoprevention by encapsulation of (-)-epigallocatechin-3-gallate with bioactive peptides/chitosan nanoparticles for enhancement of its bioavailability. Chemical Communications, 48 , 2421.

Hu, B., Xie, M., Zhang, C., \& Zeng, X. (2014). Genipin-structured peptide-polysaccharide nanoparticles with significantly improved resistance to harsh gastrointestinal environments and their potential for oral delivery of polyphenols. Journal of Agricultural and Food Chemistry, 62, 12443-12452.

Jain, S., Jain, A. K., Pohekar, M., \& Thanki, K. (2013). Novel self-emulsifying formulation of quercetin for improved in vivo antioxidant potential: Implications for drug-induced cardiotoxicity and nephrotoxicity. Free Radical Biology and Medicine, 65, 117-130.

Jin, X., Zhang, S.-b., Li, S.-m., Liang, K., \& Jia, Z.-y (2016). Influence of chitosan nanoparticles as the absorption enhancers on salvianolic acid B In vitro and In vivo evaluation. Pharmacognosy Magazine, 12, 57-63.

Joye, I. J., Davidov-Pardo, G., \& McClements, D. J. (2014). Nanotechnology for increased micronutrient bioavailability. Trends in Food Science \& Technology, 40, 168-182.

Jung, K.-H., Lee, J. H., Park, J. W., Quach, C. H. T., Moon, S.-H., Cho, Y. S., et al. (2015) Resveratrol-loaded polymeric nanoparticles suppress glucose metabolism and tumor growth in vitro and in vivo. International Journal of Pharmaceutics, 478, 251-257.

Kakkar, V., Singh, S., Singla, D., Sahwney, S., Chauhan, A. S., Singh, G., et al. (2010). Pharmacokinetic applicability of a validated liquid chromatography tandem mass spectroscopy method for orally administered curcumin loaded solid lipid nanoparticles to rats. Journal of Chromatography B, 878, 3427-3431.

Katouzian, I., Faridi Esfanjani, A., Jafari, S. M., \& Akhavan, S. (2017). Formulation and application of a new generation of lipid nano-carriers for the food bioactive ingredients. Trends in Food Science \& Technology, 68, 14-25.

Katouzian, I., \& Jafari, S. M. (2016). Nano-encapsulation as a promising approach for targeted delivery and controlled release of vitamins. Trends in Food Science \& Technology, 53, 34-48.

Kesarwani, K., \& Gupta, R. (2013). Bioavailability enhancers of herbal origin: An overview. Asian Pacific Journal of Tropical Biomedicine, 3, 253-266.

Kettiger, H., Schipanski, A., Wick, P., \& Huwyler, J. (2013). Engineered nanomaterial uptake and tissue distribution: From cell to organism. International Journal of Nanomedicine, 3255-3269.

Khalil, N. M., Nascimento, T. C. F, d., Casa, D. M., Dalmolin, L. F., Mattos, A. C. d., Hoss, I., et al. (2013). Pharmacokinetics of curcumin-loaded PLGA and PLGA-PEG blend nanoparticles after oral administration in rats. Colloids and Surfaces B: Biointerfaces, $101,353-360$.

Klang, V., \& Valenta, C. (2011). Lecithin-based nanoemulsions. Journal of Drug Delivery Science and Technology, 21, 55-76.

Komaiko, J., Sastrosubroto, A., \& McClements, D. J. (2016). Encapsulation of $\omega-3$ fatty acids in nanoemulsion-based delivery systems fabricated from natural emulsifiers: Sunflower phospholipids. Food Chemistry, 203, 331-339.

Kostewicz, E. S., Abrahamsson, B., Brewster, M., Brouwers, J., Butler, J., Carlert, S., et al. (2014). In vitro models for the prediction of in vivo performance of oral dosage forms. European Journal of Pharmaceutical Sciences, 57, 342-366.

Krug, S. M., Amasheh, M., Dittmann, I., Christoffel, I., Fromm, M., \& Amasheh, S. (2013). Sodium caprate as an enhancer of macromolecule permeation across tricellular tight junctions of intestinal cells. Biomaterials, 34, 275-282.

Lai, D. Y. (2012). Toward toxicity testing of nanomaterials in the 21st century: A paradigm for moving forward. Wiley Interdisciplinary Reviews: Nanomedicine and Nanobiotechnology, 4, 1-15.

Lai, S. K., Wang, Y.-Y., \& Hanes, J. (2009). Mucus-penetrating nanoparticles for drug and gene delivery to mucosal tissues. Advanced Drug Delivery Reviews, 61, 158-171.

Lambert, J. D., Kwon, S.-J., Ju, J., Bose, M., Lee, M.-J., Hong, J., et al. (2008). Effect of genistein on the bioavailability and intestinal cancer chemopreventive activity of (-)-epigallocatechin-3-gallate. Carcinogenesis, 29, 2019-2024.

Laparra, J. M., \& Sanz, Y. (2010). Interactions of gut microbiota with functional food components and nutraceuticals. Pharmacological Research, 61, 219-225.

Lasoń, E., Sikora, E., Ogonowski, J., Tabaszewska, M., \& Skoczylas, Ł. (2016). Release study of selected terpenes from nanostructured lipid carriers. Colloids and Surfaces A: Physicochemical and Engineering Aspects, 510, 87-92.

Lee, C.-W., Yen, F.-L., Huang, H.-W., Wu, T.-H., Ko, H.-H., Tzeng, W.-S., et al. (2012). Resveratrol nanoparticle system improves dissolution properties and enhances the hepatoprotective effect of resveratrol through antioxidant and anti-inflammatory pathways. Journal of Agricultural and Food Chemistry, 60, 4662-4671.

Leonard, N. B. (2000). Stability testing of nutraceuticals and functional foods. Handbook of nutraceuticals and functional foods. CRC Press.

Lerner, A., \& Matthias, T. (2015). Changes in intestinal tight junction permeability associated with industrial food additives explain the rising incidence of autoimmune disease. Autoimmunity Reviews, 14, 479-489.

Li, Z., \& Gu, L. (2014). Fabrication of self-assembled (-)-Epigallocatechin gallate (EGCG) ovalbumin-dextran conjugate nanoparticles and their transport across monolayers of human intestinal epithelial Caco-2 cells. Journal of Agricultural and Food Chemistry, 62, 1301-1309.

Li, Z., Jiang, H., Xu, C., \& Gu, L. (2015a). A review: Using nanoparticles to enhance absorption and bioavailability of phenolic phytochemicals. Food Hydrocolloids, 43, 153-164.

Lindahl, A., Ungell, A., Knutson, L., \& Lennernäs, H. (1997). Characterization of fluids from the stomach and proximal jejunum in men and women. Pharmaceutical Research, $14,497-502$.

Li, L., Ni, R., Shao, Y., \& Mao, S. (2014). Carrageenan and its applications in drug delivery. Carbohydrate Polymers, 103, 1-11.

Li, Z., Paulson, A. T., \& Gill, T. A. (2015b). Encapsulation of bioactive salmon protein hydrolysates with chitosan-coated liposomes. Journal of Functional Foods, 19, 733-743.

Liu, W., Pan, H., Zhang, C., Zhao, L., Zhao, R., Zhu, Y., et al. (2016). Developments in methods for measuring the intestinal absorption of nanoparticle-bound drugs. International Journal of Molecular Sciences, 17, 1171.

Livney, Y. D. (2015). Nanostructured delivery systems in food: Latest developments and potential future directions. Current Opinion in Food Science, 3, 125-135.

Li, W., Yi, S., Wang, Z., Chen, S., Xin, S., Xie, J., et al. (2011). Self-nanoemulsifying drug delivery system of persimmon leaf extract: Optimization and bioavailability studies. International Journal of Pharmaceutics, 420, 161-171.

Lopes, M., Shrestha, N., Correia, A., Shahbazi, M.-A., Sarmento, B., Hirvonen, J., et al. (2016). Dual chitosan/albumin-coated alginate/dextran sulfate nanoparticles for enhanced oral delivery of insulin. Journal of Controlled Release, 232, 29-41.

Lorkowski, G. (2012). Gastrointestinal absorption and biological activities of serine and 
cysteine proteases of animal and plant origin: Review on absorption of serine and cysteine proteases. International Journal of Physiology, Pathophysiology and Pharmacology, 4, 10-27.

Lotito, S. B., Zhang, W.-J., Yang, C. S., Crozier, A., \& Frei, B. (2011). Metabolic conversion of dietary flavonoids alters their anti-inflammatory and antioxidant properties. Free Radical Biology and Medicine, 51, 454-463.

Lucarini, S., Fagioli, L., Campana, R., Cole, H., Duranti, A., Baffone, W., et al. (2016). Unsaturated fatty acids lactose esters: Cytotoxicity, permeability enhancement and antimicrobial activity. European Journal of Pharmaceutics and Biopharmaceutics, 107, $88-96$.

Luo, Z., Liu, Y., Zhao, B., Tang, M., Dong, H., Zhang, L., et al. (2013). Ex vivo and in situ approaches used to study intestinal absorption. Journal of Pharmacological and Toxicological Methods, 68, 208-216.

Madalena, D. A., Ramos, Ó. L., Pereira, R. N., Bourbon, A. I., Pinheiro, A. C., Malcata, F. $X$., et al. (2016). In vitro digestion and stability assessment of $\beta$-lactoglobulin/riboflavin nanostructures. Food Hydrocolloids, 58, 89-97.

Maher, S., Mrsny, R. J., \& Brayden, D. J. (2016). Intestinal permeation enhancers for oral peptide delivery. Advanced Drug Delivery Reviews, 106, 277-319.

Mansuri, S., Kesharwani, P., Jain, K., Tekade, R. K., \& Jain, N. K. (2016). Mucoadhesion: A promising approach in drug delivery system. Reactive and Functional Polymers, 100, 151-172.

Martins, J. T., Santos, S. F., Bourbon, A. I., Pinheiro, A. C., González-Fernández, Á., Pastrana, L. M., et al. (2016). Lactoferrin-based nanoparticles as a vehicle for iron in food applications - development and release profile. Food Research International, 90, $16-24$.

McCartney, F., Gleeson, J. P., \& Brayden, D. J. (2016). Safety concerns over the use of intestinal permeation enhancers: A mini-review. Tissue Barriers, 4, e1176822.

McClements, D. J. (2012a). Requirements for food ingredient and nutraceutical delivery systems. Encapsulation technologies and delivery systems for food ingredients and nutraceuticals (pp. 3-18). Woodhead Publishing.

McClements, D. J. (2012b). Nanoemulsions versus microemulsions: Terminology, differences, and similarities. Soft Matter, 8, 1719-1729.

McClements, D. J. (2014a). Key physicochemical Concepts. Nanoparticle- and microparticle-based delivery Systems: Encapsulation, protection and release of active compoundsBoca Raton: CRC Press 10.

McClements, D. J. (2014b). Background and context. Nanoparticle- and microparticle-based delivery systems (pp. 1-36). CRC Press.

McClements, D. J. (2015a). Enhancing nutraceutical bioavailability through food matrix design. Current Opinion in Food Science, 4, 1-6.

McClements, D. J. (2015b). Nanoscale nutrient delivery systems for food Applications: Improving bioactive dispersibility, stability, and bioavailability. Journal of Food Science, 80, N1602-N1611.

McClements, D. J., Decker, E. A., \& Park, Y. (2008). Controlling lipid bioavailability through physicochemical and structural approaches. Critical Reviews in Food Science and Nutrition, 49, 48-67.

McClements, D. J., Decker, E. A., Park, Y., \& Weiss, J. (2009). Structural design principles for delivery of bioactive components in nutraceuticals and functional foods. Critical Reviews in Food Science and Nutrition, 49, 577-606.

McClements, D. J., \& Li, Y. (2010). Structured emulsion-based delivery systems: Controlling the digestion and release of lipophilic food components. Advances in Colloid and Interface Science, 159, 213-228.

McClements, D. J., Li, F., \& Xiao, H. (2015c). The nutraceutical bioavailability classification Scheme: Classifying nutraceuticals according to factors limiting their oral bioavailability. Annual Review of Food Science and Technology, 6, 299-327.

McClements, D. J., Saliva-Trujillo, L., Zhang, R., Zhang, Z., Zou, L., Yao, M., et al. (2016). Boosting the bioavailability of hydrophobic nutrients, vitamins, and nutraceuticals in natural products using excipient emulsions. Food Research International, 88, 140-152.

McClements, D. J., \& Xiao, H. (2014). Excipient foods: Designing food matrices that improve the oral bioavailability of pharmaceuticals and nutraceuticals. Food \& Function, 5, 1320-1333.

McClements, D. J., Zou, L., Zhang, R., Salvia-Trujillo, L., Kumosani, T., \& Xiao, H. (2015d). Enhancing nutraceutical performance using excipient Foods: Designing food structures and compositions to increase bioavailability. Comprehensive Reviews in Food Science and Food Safety, 14, 824-847.

Mehrad, B., Ravanfar, R., Licker, J., Regenstein, J. M., \& Abbaspourrad, A. (2018). Enhancing the physicochemical stability of $\beta$-carotene solid lipid nanoparticle (SLNP) using whey protein isolate. Food Research International, 105, 962-969.

Misaka, S., Müller, F., \& Fromm, M. F. (2013). Clinical relevance of drug efflux pumps in the gut. Current Opinion in Pharmacology, 13, 847-852.

Moghimipour, E., Ameri, A., \& Handali, S. (2015). Absorption-enhancing effects of bile salts. Molecules, 20, 14451

Mokhtari, S., Jafari, S. M., \& Assadpour, E. (2017). Development of a nutraceutical nanodelivery system through emulsification/internal gelation of alginate. Food Chemistry, 229, 286-295.

Montes de Oca-Ávalos, J. M., Candal, R. J., \& Herrera, M. L. (2017). Colloidal properties of sodium caseinate-stabilized nanoemulsions prepared by a combination of a highenergy homogenization and evaporative ripening methods. Food Research International, 100, 143-150.

Müller, C., Leithner, K., Hauptstein, S., Hintzen, F., Salvenmoser, W., \& BernkopSchnürch, A. (2012). Preparation and characterization of mucus-penetrating papain/ poly(acrylic acid) nanoparticles for oral drug delivery applications. Journal of Nanoparticle Research, 15, 1-13.

Nasr, M. (2016). Development of an optimized hyaluronic acid-based lipidic nanoemulsion co-encapsulating two polyphenols for nose to brain delivery. Drug Delivery, 23, 1444-1452.

Noh, J., Kim, J., Kim, J. S., Chung, Y. S., Chang, S. T., \& Park, J. (2018).
Microencapsulation by pectin for multi-components carriers bearing both hydrophobic and hydrophilic active agents. Carbohydrate Polymers, 182, 172-179.

Oehlke, K., Adamiuk, M., Behsnilian, D., Graf, V., Mayer-Miebach, E., Walz, E., et al. (2014). Potential bioavailability enhancement of bioactive compounds using foodgrade engineered nanomaterials: A review of the existing evidence. Food Funct, 5, 1341-1359.

Okada, T., Mizuno, Y., Sibayama, S., Hosokawa, M., \& Miyashita, K. (2011). Antiobesity effects of Undaria lipid Capsules prepared with Scallop phospholipids. Journal of Food Science, 76, H2-H6.

Ozdal, T., Sela, D. A., Xiao, J., Boyacioglu, D., Chen, F., \& Capanoglu, E. (2016). The reciprocal interactions between polyphenols and gut microbiota and effects on bioaccessibility. Nutrients, 8,78 .

Panyoyai, N., Shanks, R. A., \& Kasapis, S. (2017). Tocopheryl acetate release from mi crocapsules of waxy maize starch. Carbohydrate Polymers, 167, 27-35.

Paques, J. P., van der Linden, E., van Rijn, C. J. M., \& Sagis, L. M. C. (2014). Preparation methods of alginate nanoparticles. Advances in Colloid and Interface Science, 209, 163-171.

Park, M. S., Chang, J. H., \& Hoboken (2011). Absorption of drugs via passive diffusion and Carrier-Mediated pathways. In M. Hu, \& X. Li (Eds.). Oral Bioavailability: Basic principles, advanced Concepts, and applications (pp. 63-75). Hoboken, NJ, USA: : John Wiley \& Sons, Inc.

Parthasarathi, S., Muthukumar, S. P., \& Anandharamakrishnan, C. (2016). The influence of droplet size on the stability, in vivo digestion, and oral bioavailability of vitamin $\mathrm{E}$ emulsions. Food \& Function, 7, 2294-2302.

Pathak, K., \& Raghuvanshi, S. (2015). Oral bioavailability: Issues and solutions via nanoformulations. Clinical Pharmacokinetics, 54, 325-357.

Paul, A., Das, S., Das, J., Samadder, A., \& Khuda-Bukhsh, A. R. (2013). Cytotoxicity and apoptotic signalling cascade induced by chelidonine-loaded PLGA nanoparticles in HepG2 cells in vitro and bioavailability of nano-chelidonine in mice in vivo. Toxicology Letters, 222, 10-22.

Penalva, R., González-Navarro, C. J., Gamazo, C., Esparza, I., \& Irache, J. M. (2017). Zein nanoparticles for oral delivery of quercetin: Pharmacokinetic studies and preventive anti-inflammatory effects in a mouse model of endotoxemia. Nanomedicine: Nanotechnology, Biology and Medicine, 13, 103-110.

Peppas, N. A., Bures, P., Leobandung, W., \& Ichikawa, H. (2000). Hydrogels in pharmaceutical formulations. European Journal of Pharmaceutics and Biopharmaceutics, 50, $27-46$.

Pereira de Sousa, I., Cattoz, B., Wilcox, M. D., Griffiths, P. C., Dalgliesh, R., Rogers, S., et al. (2015). Nanoparticles decorated with proteolytic enzymes, a promising strategy to overcome the mucus barrier. European Journal of Pharmaceutics and Biopharmaceutics, 97, Part a, 257-264.

Perumal, V., Manickam, T., Bang, K.-S., Velmurugan, P., \& Oh, B.-T. (2016). Antidiabetic potential of bioactive molecules coated chitosan nanoparticles in experimental rats. International Journal of Biological Macromolecules, 92, 63-69.

Pinheiro, A. C., Bourbon, A. I., Cerqueira, M. A., Maricato, E., Nunes, C., Coimbra, M. A. et al. (2015). Chitosan/fucoidan multilayer nanocapsules as a vehicle for controlled release of bioactive compounds. Carbohydrate Polymers, 115, 1-9.

Pinheiro, A. C., Coimbra, M. A., \& Vicente, A. A. (2016). In vitro behaviour of curcumin nanoemulsions stabilized by biopolymer emulsifiers - effect of interfacial composition. Food Hydrocolloids, 52, 460-467.

Pinheiro, A. C., Gonçalves, R. F. S., Madalena, D. A., \& Vicente, A. A. (2017). Towards the understanding of the behavior of bio-based nanostructures during in vitro digestion. Current Opinion in Food Science, 15, 79-86.

Pinheiro, A. C., Lad, M., Silva, H. D., Coimbra, M. A., Boland, M., \& Vicente, A. A. (2013) Unravelling the behaviour of curcumin nanoemulsions during in vitro digestion: Effect of the surface charge. Soft Matter, 9, 3147.

Piperigkou, Z., Karamanou, K., Engin, A. B., Gialeli, C., Docea, A. O., Vynios, D. H., et al (2016). Emerging aspects of nanotoxicology in health and disease: From agriculture and food sector to cancer therapeutics. Food and Chemical Toxicology, 91, 42-57.

Plapied, L., Duhem, N., des Rieux, A., \& Préat, V. (2011). Fate of polymeric nanocarriers for oral drug delivery. Current Opinion in Colloid \& Interface Science, 16, 228-237.

Podila, R., \& Brown, J. M. (2013). Toxicity of engineered nanomaterials: A physicochemical perspective. Journal of Biochemical and Molecular Toxicology, 27, 50-55.

Porter, C. J., Trevaskis, N. L., \& Charman, W. N. (2007). Lipids and lipid-based formulations: Optimizing the oral delivery of lipophilic drugs. Nature Reviews Drug Discovery, 6, 231-248.

Qazi, G. N. C. T. L., Gupta, A. K. S.,G. K., Gupta, D. K., Jaggi, B. S., et al. (2003). Bioavailability enhancing activity of zingiber officinale linn and its extracts/fractions thereof. In: Google Patents.

Qian, C., Decker, E. A., Xiao, H., \& McClements, D. J. (2012). Nanoemulsion delivery systems: Influence of Carrier oil on $\beta$-carotene bioaccessibility. Food Chemistry, 135 $1440-1447$.

Ramalingam, P., \& Ko, Y. T. (2016). Improved oral delivery of resveratrol from N-trimethyl chitosan-g-palmitic acid surface-modified solid lipid nanoparticles. Colloids and Surfaces B: Biointerfaces, 139, 52-61.

Ramteke, K. H., Joshi, S. A., \& Dhole, S. N. (2012). Solid lipid nanoparticle: A review. IOSR Journal of Pharmacy, 2, 34-44.

Rao, J., \& McClements, D. J. (2011). Food-grade microemulsions, nanoemulsions and emulsions: Fabrication from sucrose monopalmitate \& lemon oil. Food Hydrocolloids, $25,1413-1423$.

Rastogi, H., \& Jana, S. (2014). Evaluation of physicochemical properties and intestina permeability of six dietary polyphenols in human intestinal colon adenocarcinoma Caco-2 cells. European Journal of Drug Metabolism and Pharmacokinetics, 41, 33-43.

Rein, M. J., Renouf, M., Cruz-Hernandez, C., Actis-Goretta, L., Thakkar, S. K., \& da Silva Pinto, M. (2013). Bioavailability of bioactive food compounds: A challenging journey to bioefficacy. British Journal of Clinical Pharmacology, 75, 588-602. 
Renukuntla, J., Vadlapudi, A. D., Patel, A., Boddu, S. H. S., \& Mitra, A. K. (2013). Approaches for enhancing oral bioavailability of peptides and proteins. International Journal of Pharmaceutics, 447, 75-93.

des Rieux, A., Fievez, V., Théate, I., Mast, J., Préat, V., \& Schneider, Y.-J. (2007). An improved in vitro model of human intestinal follicle-associated epithelium to study nanoparticle transport by M cells. European Journal of Pharmaceutical Sciences, 30, 380-391.

Rossi, M., Cubadda, F., Dini, L., Terranova, M. L., Aureli, F., Sorbo, A., et al. (2014). Scientific basis of nanotechnology, implications for the food sector and future trends. Trends in Food Science \& Technology, 40, 127-148.

Salvia-Trujillo, L., \& McClements, D. J. (2016). Enhancement of lycopene bioaccessibility from tomato juice using excipient emulsions: Influence of lipid droplet size. Food Chemistry, 210, 295-304.

Salvia-Trujillo, L., Qian, C., Martín-Belloso, O., \& McClements, D. J. (2013). Influence of particle size on lipid digestion and $\beta$-carotene bioaccessibility in emulsions and nanoemulsions. Food Chemistry, 141, 1472-1480.

Schimpel, C., Teubl, B., Absenger, M., Meindl, C., Fröhlich, E., Leitinger, G., et al. (2014). Development of an advanced intestinal in vitro triple culture permeability model to study transport of nanoparticles. Molecular Pharmaceutics, 11, 808-818.

Shafaei, A., Esmailli, K., Farsi, E., Aisha, A. F. A., Abul Majid, A. M. S., \& Ismail, Z. (2015). Genotoxicity, acute and subchronic toxicity studies of nano liposomes of Orthosiphon stamineus ethanolic extract in Sprague Dawley rats. BMC Complementary and Alternative Medicine, 15.

Shahbazi, M.-A., \& Santos, H. A. (2013). Improving oral absorption via drug-loaded Nanocarriers: Absorption mechanisms, intestinal models and rational fabrication. Current Drug Metabolism, 14, 23-56.

Shahidi, F., \& Zhong, Y. (2008). Bioactive peptides. Journal of AOAC International, 91, 914-931.

Shaikh, J., Ankola, D. D., Beniwal, V., Singh, D., \& Kumar, M. N. V. R. (2009). Nanoparticle encapsulation improves oral bioavailability of curcumin by at least 9 fold when compared to curcumin administered with piperine as absorption enhancer. European Journal of Pharmaceutical Sciences, 37, 223-230.

Shin, G. H., Kim, J. T., \& Park, H. J. (2015). Recent developments in nanoformulations of lipophilic functional foods. Trends in Food Science \& Technology, 46, 144-157.

Shoba, G., Joy, D., Joseph, T., Majeed, M., Rajendran, R., \& Srinivas, P. S. S. R. (1998) Influence of piperine on the pharmacokinetics of curcumin in animals and human volunteers. Planta Medica, 64, 353-356.

Shutava, T. G., Balkundi, S. S., Vangala, P., Steffan, J. J., Bigelow, R. L., Cardelli, J. A., et al. (2009). Layer-by-Layer-Coated gelatin nanoparticles as a vehicle for delivery of natural polyphenols. ACS Nano, 3, 1877-1885.

Sinha, V. R., \& Kumria, R. (2003). Microbially triggered drug delivery to the colon. European Journal of Pharmaceutical Sciences, 18, 3-18.

Sjögren, E., Abrahamsson, B., Augustijns, P., Becker, D., Bolger, M. B., Brewster, M., et al. (2014). In vivo methods for drug absorption - Comparative physiologies, model selection, correlations with in vitro methods (IVIVC), and applications for formulation/ API/excipient characterization including food effects. European Journal of Pharmaceutical Sciences, 57, 99-151.

Sonaje, K., Chuang, E.-Y., Lin, K.-J., Yen, T.-C., Su, F.-Y., Tseng, M. T., et al. (2012). Opening of epithelial tight junctions and enhancement of paracellular permeation by Chitosan: Microscopic, ultrastructural, and computed-tomographic observations. Molecular Pharmaceutics, 9, 1271-1279.

Song, Q., Wang, X., Hu, Q., Huang, M., Yao, L., Qi, H., et al. (2013). Cellular internalization pathway and transcellular transport of pegylated polyester nanoparticles in Caco-2 cells. International Journal of Pharmaceutics, 445, 58-68.

Sotelo-Boyás, M. E., Correa-Pacheco, Z. N., Bautista-Baños, S., \& Corona-Rangel, M. L. (2017). Physicochemical characterization of chitosan nanoparticles and nanocapsules incorporated with lime essential oil and their antibacterial activity against food borne pathogens. LWT - Food Science and Technology, 77, 15-20.

Souza, M. P., Vaz, A. F. M., Costa, T. B., Cerqueira, M. A., De Castro, C. M. M. B., Vicente, A. A., et al. (2018). Construction of a biocompatible and antioxidant multilayer coating by layer-by-layer assembly of $\mathrm{\kappa}$-carrageenan and quercetin nanoparticles Food and Bioprocess Technology, 11, 1050-1060.

Sun, F., Ju, C., Chen, J., Liu, S., Liu, N., Wang, K., et al. (2011). Nanoparticles based on hydrophobic alginate derivative as nutraceutical delivery Vehicle: Vitamin D3Loading. Artificial Cells, Blood Substitutes, and Biotechnology, 40, 113-119.

Szúts, A., \& Szabó-Révész, P. (2012). Sucrose esters as natural surfactants in drug delivery systems-a mini-review. International Journal of Pharmaceutics, 433, 1-9.

Takahashi, M., Uechi, S., Takara, K., Asikin, Y., \& Wada, K. (2009). Evaluation of an oral Carrier system in Rats: Bioavailability and antioxidant properties of liposome-encapsulated curcumin. Journal of Agricultural and Food Chemistry, 57, 9141-9146.

Tamjidi, F., Shahedi, M., Varshosaz, J., \& Nasirpour, A. (2013). Nanostructured lipid carriers (NLC): A potential delivery system for bioactive food molecules. Innovative Food Science \& Emerging Technologies, 19, 29-43.

Tang, D.-W., Yu, S.-H., Ho, Y.-C., Huang, B.-Q., Tsai, G.-J., Hsieh, H.-Y., et al. (2013). Characterization of tea catechins-loaded nanoparticles prepared from chitosan and an edible polypeptide. Food Hydrocolloids, 30, 33-41.

Tan, B.-J., Liu, Y., Chang, K.-L., Lim, B. K., \& Chiu, G. N. C. (2012). Perorally active nanomicellar formulation of quercetin in the treatment of lung cancer. International Journal of Nanomedicine, 651.

Teng, Z., Luo, Y., Wang, T., Zhang, B., \& Wang, Q. (2013). Development and application of nanoparticles synthesized with folic acid conjugated soy protein. Journal of Agricultural and Food Chemistry, 61, 2556-2564.

Teo, A., Lee, S. J., \& Goh, K. K. T. (2017). Formation and stability of single and bi-layer nanoemulsions using WPI and lactoferrin as interfacial coatings under different environmental conditions. Food Structure, 14, 60-67.

Tian, C., Asghar, S., Wu, Y., Chen, Z., Jin, X., Yin, L., et al. (2017). Improving intestinal absorption and oral bioavailability of curcumin via taurocholic acid-modified nanostructured lipid carriers. International Journal of Nanomedicine, 12, 7897-7911.

Ting, Y., Jiang, Y., Ho, C.-T., \& Huang, Q. (2014). Common delivery systems for enhancing in vivo bioavailability and biological efficacy of nutraceuticals. Journal of Functional Foods, 7, 112-128.

Ting, Y., Zhao, Q., Xia, C., \& Huang, Q. (2015). Using in vitro and in vivo models to evaluate the oral bioavailability of nutraceuticals. Journal of Agricultural and Food Chemistry, 63, 1332-1338.

Tozaki, H., Emi, Y., Horisaka, E. R. I., Fujita, T., Yamamoto, A., \& Muranishi, S. (1997) Degradation of insulin and Calcitonin and their protection by various protease Inhibitors in rat caecal Contents: Implications in peptide delivery to the colon. Journal of Pharmacy and Pharmacology, 49, 164-168.

Tso, P. (2000). Overview of digestion and absorption. In K. D. S. Crissinger, \& M.H (Eds.) Biochemical and physiological aspects of human nutrition (pp. 75-90). Philadelphia, Pennsylvania: W.B. Saunders Company.

Vecchione, R., Quagliariello, V., Calabria, D., Calcagno, V., De Luca, E., Iaffaioli, R. V., et al. (2016). Curcumin bioavailability from oil in water nano-emulsions: In vitro and in vivo study on the dimensional, compositional and interactional dependence. Journal of Controlled Release, 233, 88-100.

de Vos, P., Faas, M. M., Spasojevic, M., \& Sikkema, J. (2010). Encapsulation for preservation of functionality and targeted delivery of bioactive food components. International Dairy Journal, 20, 292-302.

Wajda, R., Zirkel, J., \& Schaffer, T. (2007). Increase of bioavailability of coenzyme Q10 and vitamin E. Journal of Medicinal Food, 10, 731-734.

Walker, R. M., Gumus, C. E., Decker, E. A., \& McClements, D. J. (2017). Improvements in the formation and stability of fish oil-in-water nanoemulsions using Carrier oils: MCT, thyme oil, \& lemon oil. Journal of Food Engineering, 211, 60-68.

Wang, P., Heber, D., \& Henning, S. M. (2012). Quercetin increased bioavailability and decreased methylation of green tea polyphenols in vitro and in vivo. Food \& Function, $3,635-642$.

Wang, Y., Ma, Y., Zheng, Y., Song, J., Yang, X., Bi, C., et al. (2013). In vitro and in vivo anticancer activity of a novel puerarin nanosuspension against colon cancer, with high efficacy and low toxicity. International Journal of Pharmaceutics, 441, 728-735.

Yao, M., McClements, D. J., \& Xiao, H. (2015). Improving oral bioavailability of nutraceuticals by engineered nanoparticle-based delivery systems. Current Opinion in Food Science, 2, 14-19.

Yao, M., Xiao, H., \& McClements, D. J. (2014). Delivery of lipophilic bioactives: Assembly, disassembly, and reassembly of lipid nanoparticles. Annu Rev Food Sci Technol, 5, 53-81.

Yostawonkul, J., Surassmo, S., Iempridee, T., Pimtong, W., Suktham, K., Sajomsang, W. et al. (2017). Surface modification of nanostructure lipid Carrier (NLC) by oleoylquaternized-chitosan as a mucoadhesive nanocarrier. Colloids and Surfaces B: Biointerfaces, 149, 301-311.

Yuan, S., Hollinger, M., Lachowicz-Scroggins, M. E., Kerr, S. C., Dunican, E. M., Daniel, B. M., et al. (2015). Oxidation increases mucin polymer cross-links to stiffen airway mucus gels. Science Translational Medicine, 7 276ra227-276ra227.

Yu, H., \& Huang, Q. (2012). Improving the oral bioavailability of curcumin using novel organogel-based nanoemulsions. Journal of Agricultural and Food Chemistry, 60, 5373-5379.

Yun, Y., Cho, Y. W., \& Park, K. (2013). Nanoparticles for oral delivery: Targeted nanoparticles with peptidic ligands for oral protein delivery. Advanced Drug Delivery Reviews, 65, 822-832.

Yu, S.-H., Tang, D.-W., Hsieh, H.-Y., Wu, W.-S., Lin, B.-X., Chuang, E.-Y., et al. (2013). Nanoparticle-induced tight-junction opening for the transport of an anti-angiogenic sulfated polysaccharide across Caco-2 cell monolayers. Acta Biomaterialia, 9, 7449-7459.

Yu, M., Yang, Y., Zhu, C., Guo, S., \& Gan, Y. (2016). Advances in the transepithelial transport of nanoparticles. Drug Discovery Today, 21, 1155-1161.

Zhang, Y., Cui, L., Li, F., Shi, N., Li, C., Yu, X., et al. (2016b). Design, fabrication and biomedical applications of zein-based nano/micro-Carrier systems. International Journal of Pharmaceutics, 513, 191-210.

Zhang, J., Field, C. J., Vine, D., \& Chen, L. (2014). Intestinal uptake and transport of vitamin B12-loaded soy protein nanoparticles. Pharmaceutical Research, 32, $1288-1303$.

Zhang, Z.-H., Wang, X.-P., Ayman, W. Y., Munyendo, W. L. L., Lv, H.-X., \& Zhou, J.-P. (2013). Studies on lactoferrin nanoparticles of gambogic acid for oral delivery. Drug Delivery, 20, 86-93.

Zhang, X., \& Wu, W. (2014). Ligand-mediated active targeting for enhanced oral absorption. Drug Discovery Today, 19, 898-904.

Zhang, R., Zhang, Z., Kumosani, T., Khoja, S., Abualnaja, K. O., \& McClements, D. J. (2016c). Encapsulation of $\beta$-carotene in nanoemulsion-based delivery systems formed by spontaneous Emulsification: Influence of lipid composition on stability and bioaccessibility. Food Biophysics, 11, 154-164.

Zhang, R., Zhang, Z., Zou, L., Xiao, H., Zhang, G., Decker, E. A., et al. (2016a). Enhancement of carotenoid bioaccessibility from carrots using excipient emulsions: Influence of particle size of digestible lipid droplets. Food and Function, 7, 93-103.

Zimet, P., \& Livney, Y. D. (2009). Beta-lactoglobulin and its nanocomplexes with pectin as vehicles for $\omega-3$ polyunsaturated fatty acids. Food Hydrocolloids, 23, 1120-1126.

Zimet, P., Rosenberg, D., \& Livney, Y. D. (2011). Re-assembled casein micelles and casein nanoparticles as nano-vehicles for $\omega-3$ polyunsaturated fatty acids. Food Hydrocolloids, 25, 1270-1276.

Zou, L., Zheng, B., Zhang, R., Zhang, Z., Liu, W., Liu, C., et al. (2016). Enhancing the bioaccessibility of hydrophobic bioactive agents using mixed colloidal dispersions: Curcumin-loaded zein nanoparticles plus digestible lipid nanoparticles. Food Research International, 81, 74-82. 